Pacific

Journal of

Mathematics

MODULAR REPRESENTATIONS OF THE SUPERGROUP $Q(n)$ PART II

JONATHAN BRUNDAN

Volume $224 \quad$ No. 1

March 2006 


\title{
MODULAR REPRESENTATIONS OF THE SUPERGROUP $Q(n)$ PART II
}

\author{
JONATHAN BRUNDAN
}

\begin{abstract}
We continue our study of the representations of the supergroup $Q(n)$ over a field of odd positive characteristic. The focus here is on the aspects of the theory that depend in some way on the interpretation of induction in terms of sheaf cohomology of certain equivariant vector bundles on the associated flag superschemes.
\end{abstract}

\section{Introduction}

This is the second of two articles investigating the representation theory of the supergroup $G=Q(n)$ over an algebraically closed field $k$ of characteristic $p>2$. In the first article, [Brundan and Kleshchev 2003], we extended from characteristic 0 to characteristic $p$ many of the basic algebraic properties, such as the results of [Penkov 1986]. The present article is concerned instead with results that depend in some way on the interpretation of induction from a Borel subgroup $B$ in terms of sheaf cohomology on the flag superscheme $G / B$. These flag superschemes, and their analogues $G / P$ for arbitrary parabolic subgroups of $G$, were introduced originally by Manin [1997; 1991], and have already played a fundamental role in the work of Penkov and Serganova [1989; 1997a; 1997b] on the representation theory of $Q(n)$ over $\mathbb{C}$; see also [Brundan 2004].

To sketch the results proved here in more detail, recall from [Brundan and Kleshchev 2003] that the irreducible representations of $G$ are parametrized by $p$ strict dominant weights, that is, by the set

$$
X_{p}^{+}(n):=\left\{\begin{array}{l|l}
\lambda=\left(\lambda_{1}, \ldots, \lambda_{n}\right) \in \mathbb{Z}^{n} & \begin{array}{l}
\lambda_{1} \geq \cdots \geq \lambda_{n} \text { with } \\
\lambda_{i}=\lambda_{i+1} \text { only if } p \mid \lambda_{i}
\end{array}
\end{array}\right\} .
$$

The irreducible representation $L(\lambda)$ corresponding to $\lambda \in X_{p}^{+}(n)$ can be realized as the unique irreducible submodule of the induced module

$$
H^{0}(\lambda):=H^{0}(G / B, \mathscr{L}(\mathfrak{u}(\lambda))),
$$

MSC2000: 20G05.

Keywords: supergroups, flag superschemes.

Work partially supported by the NSF (grant no. DMS-0139019). 
where $\mathfrak{u}(\lambda)$ is a certain irreducible $B$-supermodule of dimension a power of 2 corresponding to the weight $\lambda$. For $\lambda \in X_{p}^{+}(n)$, we now study the higher cohomology modules

$$
H^{i}(\lambda):=H^{i}(G / P, \mathscr{L}(\mathfrak{u}(\lambda))),
$$

where $P$ is the largest parabolic subgroup that the $B$-supermodule $\mathfrak{u}(\lambda)$ can be inflated to. Let

$$
E_{\lambda}:=\sum_{i \geq 0}(-1)^{i} \operatorname{ch} H^{i}(\lambda)
$$

denote the corresponding Euler characteristic. In Theorem 4.3, we show that $E_{\lambda}$ is equal to the classical symmetric function known as Schur's $P$-function, scaled by $\operatorname{dim} \mathfrak{u}(\lambda)$. The method used to prove this goes back at least to Penkov [1988, §2.3], but we have attempted here to fill in some of the details, as promised in [Brundan 2004; Brundan and Kleshchev 2002].

The $E_{\lambda}$ 's are important because they form a natural basis for the character group of $G$. We would of course like to be able compute the decomposition numbers $d_{\lambda, \mu}$ defined from the equation

$$
E_{\lambda}=\sum_{\mu \in X_{p}^{+}(n)} d_{\lambda, \mu} L_{\mu}
$$

where

$$
L_{\mu}:=\operatorname{ch} L(\mu) .
$$

Note that $d_{\lambda, \mu} \in \mathbb{Z}, d_{\lambda, \lambda}=1$ and $d_{\lambda, \mu}=0$ unless $\mu \leq \lambda$ in the usual dominance ordering. It appears to be the case in all the examples we have computed that the $d_{\lambda, \mu}$ are always nonnegative. This is not obvious, since it can definitely happen that $H^{i}(\lambda) \neq 0$ for $i>0$, unlike the analogous situation when $G$ is a reductive algebraic group; see Example 5.3. There is one further piece of information about the decomposition numbers $d_{\lambda, \mu}$ proved in Theorem 6.3: they are zero unless $\lambda$ and $\mu$ have the same residue content, a purely combinatorial notion originally introduced by Leclerc and Thibon [1997] in their study of the canonical bases of the Fock space of type $A_{p-1}^{(2)}$ (see also [Brundan and Kleshchev 2003, §8]). We refer to this result as the linkage principle for Euler characteristics.

At the end of the article, we have included by way of further examples tables of the decomposition matrices $D=\left(d_{\lambda, \mu}\right)$ for the polynomial representations of $Q(n)$ of degree $d \leq 12$ in characteristic $p=3$ (with $n$ large). In that case, the dominant weights we are considering satisfy $\lambda_{i} \geq 0$ for all $i$, so we can represent them simply as partitions. Some calculations here were made by computer, using GAP 4.3. 


\section{Geometric interpretation of induction}

Throughout the article, we will work over a fixed algebraically closed field $k$ of characteristic $p \neq 2$. For our general conventions regarding superalgebras, supergroups and superschemes, we refer to [Brundan and Kleshchev 2003, §2] and [Manin 1997, chapter 3, §1-2 and chapter 4, §1]. We will simultaneously use geometric and functorial languages when talking about superschemes, in the spirit of [Demazure and Gabriel 1970; Jantzen 1987].

For a superscheme $X$, an $0_{X}$-supermodule means a sheaf $\mathcal{M}$ of abelian groups on the topological space underlying $X$ such that $M(U)$ has the additional structure of an $\mathrm{O}_{X}(U)$-supermodule for each open subset $U \subseteq X$. Moreover, for open subsets $U \subseteq V$, the restriction $M(V) \rightarrow M(U)$ is required to be an even supermodule homomorphism. Given superschemes $X, Y$, an $\mathscr{O}_{X}$-supermodule $\mathcal{M}$, and an $\mathbb{O}_{Y^{-}}$ supermodule $\mathcal{N}$, we will denote by $\mathcal{M} \otimes \mathcal{N}$ their "outer" tensor product, i.e., the $0_{X \times Y}$-supermodule $\operatorname{pr}_{X}^{*} \mathcal{M} \otimes_{\mathcal{O}_{X \times Y}} \operatorname{pr}_{Y}^{*} \mathcal{N}$. If $X, Y$ are noetherian and $\mathcal{M}, \mathcal{N}$ are quasicoherent, then $M \otimes \mathcal{N}$ is again quasicoherent and moreover $(M \otimes \mathcal{N})(U \times V)=$ $\mathcal{M}(U) \otimes_{k} \mathcal{N}(V)$ for affine open subsets $U \subseteq X, V \subseteq Y$.

Let $\mathfrak{m o d}_{\mathscr{O}_{X}}$ (resp. $\mathfrak{q} \mathfrak{c o h} \mathfrak{O}_{X}$ ) denote the category of $\mathfrak{O}_{X}$-supermodules (resp. quasicoherent $\mathrm{O}_{X}$-supermodules). Note we allow arbitrary (not necessarily homogeneous) morphisms: a morphism $f: \mathcal{M} \rightarrow \mathcal{N}$ of $\mathrm{O}_{X}$-supermodules satisfies

$$
f(a m)=(-1)^{\bar{f} \bar{a}} a f(m)
$$

for each $m \in \mathcal{M}(U)$, each $a \in \mathcal{O}_{X}(U)$ and each open subset $U$ of $X$. Here, $\bar{f}, \bar{a} \in \mathbb{Z}_{2}$ denote parity assuming $f$ and $a$ are homogeneous, and the formula should be interpreted by extending additively from the homogeneous case if they are not. The categories $\mathfrak{m o d}_{\mathscr{O}_{X}}$ and $\mathfrak{q} \mathfrak{c o h} \mathfrak{O}_{X}$ are not abelian categories, but the underlying even categories consisting of the same objects and only even morphisms are. This allows us to make use of all the usual machinery of homological algebra. We also have the parity change functor

$$
\Pi: \mathfrak{m o d}_{\mathfrak{O}_{X}} \rightarrow \mathfrak{m o d}_{\mathscr{O}_{X}}
$$

defined on objects by letting $(\Pi \mathcal{M})(U)$ equal $\mathcal{M}(U)$ (for $U \subseteq X$ open) as an abelian group, but with the opposite $\mathbb{Z}_{2}$-grading and new $\mathcal{O}_{X}(U)$-action defined by $a \cdot m:=$ $(-1)^{\bar{a}}$ am.

We have the usual sheaf cohomology functors $H^{i}(X, ?)$ from the category of sheaves of abelian groups on the underlying topological space to the category of abelian groups. If $\mathcal{M}$ is an $\mathrm{O}_{X}$-supermodule, each $H^{i}(X, \mathcal{M})$ has a canonical structure as an $O_{X}(X)$-supermodule (hence in particular as a vector superspace). This follows because the category $\mathfrak{m o d}_{\mathscr{O}_{X}}$ has enough injectives and, as in [Hartshorne 
1977, III.2.4], injective $0_{X}$-supermodules are acyclic for $H^{0}(X, ?)$; so we can compute $H^{i}(X, M)$ using an resolution of $\mathcal{M}$ by injective $\mathrm{O}_{X}$-supermodules. We recall [Hartshorne 1977, III.2.7]:

Grothendieck's vanishing theorem. For a noetherian superscheme $X$, we have $H^{i}(X, ?)=0$ for all $i>\operatorname{dim} X$, where $\operatorname{dim}$ denotes dimension as a topological space.

To generalize other basic results, one can exploit the canonical filtration of an $O_{X}$-supermodule $\mathcal{M}$, namely, the filtration

$$
M \supseteq \mathscr{F}_{X} M \supseteq \mathscr{F}_{X}^{2} M \supseteq \ldots
$$

Here, $\mathscr{F}_{X}$ denotes the quasicoherent sheaf of superideals on $X$ defined as the sheaf associated to the presheaf $U \mapsto \mathcal{O}_{X}(U) \mathscr{O}_{X}(U)_{\overline{1}}$. The underlying purely even scheme $X_{\mathrm{ev}}$ is the scheme over $k$ equal to $X$ as a topological space, with structure sheaf $\mathcal{O}_{X} / \mathscr{F}_{X}$. The factors $\mathscr{F}_{X}^{i} \mathcal{M} / \mathscr{F}_{X}^{i+1} \mathcal{M}$ in the canonical filtration are $\mathcal{O}_{X_{\mathrm{ev}}}$-modules, so since

$$
H^{i}\left(X, \mathscr{F}_{X}^{i} \mathcal{M} / \mathscr{F}_{X}^{i+1} \mathcal{M}\right)=H^{i}\left(X_{\mathrm{ev}}, \mathscr{F}_{X}^{i} \mathcal{M} / \mathscr{F}_{X}^{i+1} \mathcal{M}\right)
$$

as the underlying topological spaces of $X$ and $X_{\mathrm{ev}}$ are equal, we can obtain information about $H^{i}(X, \mathcal{M})$ using the purely even theory, the long exact sequence of cohomology and induction on the length of the canonical filtration (which is always finite for noetherian $X$ ). In particular, using [Hartshorne 1977, III.3.5 and III.5.2], one obtains:

Serre's vanishing theorem. For a noetherian superscheme $X$ with $X_{\mathrm{ev}}$ affine, we have $H^{i}(X, M)=0$ for all quasicoherent $\mathcal{O}_{X}$-supermodules $\mathcal{M}$ and all $i>0$.

Serre's finiteness theorem. For a noetherian superscheme $X$ with $X_{\mathrm{ev}}$ projective, $H^{i}(X, M)$ is finite-dimensional for all coherent $O_{X}$-supermodules $M$ and all $i \geq 0$.

We say that $X$ is decomposable if $O_{X}$ is isomorphic to the symmetric superalgebra of the $O_{X_{\mathrm{ev}}}$-supermodule $\mathscr{F}_{X} / \mathscr{F}_{X}^{2}$. If instead $X$ has an open cover $\left(U_{i}\right)_{i \in I}$ such that each $\left(U_{i},\left.\mathscr{O}_{X}\right|_{U_{i}}\right)$ is decomposable, then $X$ is called locally decomposable.

Lemma 2.1. If $X$ is locally decomposable and $\mathcal{M}$ is a locally free $O_{X}$-supermodule, there is a natural isomorphism

$$
\mathscr{F}_{X}^{i} \mathcal{M} \mathscr{F}_{X}^{i+1} \mathcal{M} \simeq S^{i}\left(\mathscr{F}_{X} / \mathscr{F}_{X}^{2}\right) \otimes_{\mathscr{O}_{\mathrm{ev}}} \mathcal{M} / \mathscr{F}_{X} \mathcal{M} .
$$

Proof. Without any assumptions on $X$ or $\mathcal{M}$, there is a natural map

$$
S^{i}\left(\mathscr{F}_{X} / \mathscr{F}_{X}^{2}\right) \otimes \sigma_{X_{\mathrm{ev}}} \mu / \mathscr{F}_{X} \mu \longrightarrow \mathscr{F}_{X}^{i} \mu / \mathscr{F}_{X}^{i+1} \mu
$$

induced by multiplication. To check that it is an isomorphism for $\mathcal{M}$ locally free and $X$ locally decomposable, reduce to the case that $X$ is affine and decomposable and $\mathcal{M}$ is free, and then argue directly. 
Now let $G$ be an algebraic supergroup in the sense of [Brundan and Kleshchev $2003, \S 2]$. Thus $G$ is a functor from the category of commutative superalgebras to the category of groups which is an affine superscheme when viewed just as a functor to sets, such that in addition the coordinate ring $k[G]$ is finitely generated as a $k$-superalgebra. Suppose we are given a right action $\rho: X \times G \rightarrow X$ of $G$ on a noetherian superscheme $X$. There is a standard notion of a $G$-equivariant $0_{X^{-}}$ supermodule ( $\mathrm{O}_{X} G$-supermodule for short), for example as in [Chriss and Ginzburg 1997, 5.1.6] or [Mumford and Fogarty 1982, 1.6]. We present here a somewhat different formulation of the definition in the quasicoherent case, which we found easier to work with.

Let $\mu: G \times G \rightarrow G$ be multiplication, $e: \operatorname{Spec} k \rightarrow G$ be the identity. The comorphisms are denoted

$$
\mu^{\#}: \mathscr{O}_{G} \rightarrow \mu_{*}\left(\mathscr{O}_{G} \otimes \mathscr{O}_{G}\right) \text { and } e^{\#}: \mathscr{O}_{G} \rightarrow e_{*} k
$$

A quasicoherent $\mathrm{O}_{X} G$-supermodule means a quasicoherent $\mathrm{O}_{X}$-supermodule $\mathcal{M}$ equipped with an even $\mathcal{O}_{X}$-supermodule map $\eta: \mathcal{M} \rightarrow \rho_{*}\left(\mathcal{M} \otimes \mathscr{O}_{G}\right)$ such that the following diagrams of sheaves on $X$ commute:
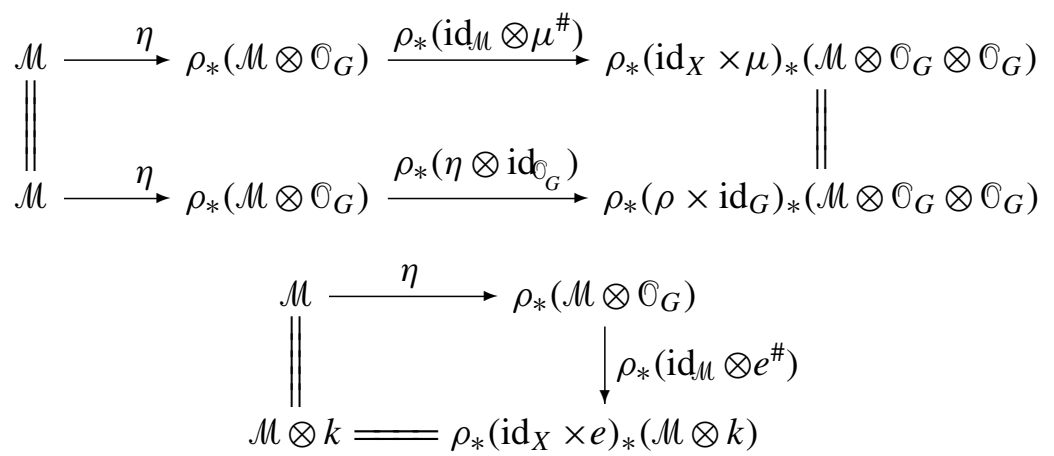

(If $X$ is a point these are just the usual comodule axioms.)

There is a functor $\operatorname{Spec}_{X}$ which defines a contravariant equivalence from the category of quasicoherent $O_{X}$-superalgebras to the category of superschemes over $X$ which are affine over $X$. The definition is the same as in the purely even case; see for example [Demazure and Gabriel 1970, I, §2, no. 3]. For a quasicoherent $\mathrm{O}_{X}$-supermodule $M$, we can consider the associated fibration

$$
\mathbb{V}(\mathcal{M}):=\operatorname{Spec}_{X} S(\mathcal{M}) \stackrel{\sigma}{\longrightarrow} X,
$$

where $S(\mathcal{M})$ denotes the symmetric superalgebra. If $\mathcal{M}$ is a quasicoherent ${ }^{\circ}{ }_{X} G$ supermodule, the structure map $\eta$ extends in a unique way to an $\mathbb{O}_{X}$-superalgebra map

$$
\tilde{\eta}: S(\mathcal{M}) \rightarrow \rho_{*}\left(S(\mathcal{M}) \otimes \mathcal{O}_{G}\right) .
$$


This in turn defines a morphism $\operatorname{Spec}_{X}\left(\rho_{*}\left(S(\mathcal{M}) \otimes O_{G}\right)\right) \rightarrow \operatorname{Spec}_{X}(S(\mathcal{M}))$ of superschemes over $X$. Composing with the canonical map

$$
\mathbb{V}(\mathcal{M}) \times G \cong \operatorname{Spec}_{X \times G}\left(S(\mathcal{M}) \otimes \mathcal{O}_{G}\right) \rightarrow \operatorname{Spec}_{X}\left(\rho_{*}\left(S(\mathcal{M}) \otimes \mathcal{O}_{G}\right)\right),
$$

we obtain a commutative diagram

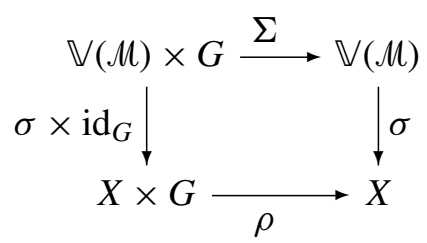

Now (3) implies that $\Sigma$ satisfies associativity; that is, there is a commutative cube analogous to that on [Mumford and Fogarty 1982, p. 31], while (4) gives that $\Sigma$ is unital in the obvious sense. So, $\Sigma$ is a "linear" right action of $G$ on the fibration $\mathbb{V}(\mathcal{M})$. Conversely, we can recover the original structure map $\eta$ of $\mathcal{M}$ from such an action $\Sigma$ since $\tilde{\eta}=\sigma_{*} \Sigma^{\#}$.

There is a natural notion of morphism between two quasicoherent $0_{X} G$-supermodules, giving us the category $\mathfrak{q} \mathfrak{c o h}_{O_{X} G}$. Its underlying even category is abelian, and moreover:

Lemma 2.2. (i) The category $\mathfrak{q} \mathfrak{c o h}_{O_{X} G}$ has enough injectives.

(ii) Injective objects in $\mathfrak{q c o h}_{\mathscr{O}_{X} G}$ are acyclic for $H^{0}(X, ?)$.

Proof. (i) One first checks that the forgetful functor res: $\mathfrak{q c o h}_{\mathbb{O}_{X} G} \rightarrow \mathfrak{q c o h} \mathfrak{O}_{\mathcal{O}_{X}}$ has a right adjoint, namely, the functor ind $:=\rho_{*}\left(? \otimes \mathcal{O}_{G}\right)$. Here for any quasicoherent $O_{X}$-supermodule $\mathcal{M}$, ind $\mathcal{M}=\rho_{*}\left(M \otimes \mathcal{O}_{G}\right)$ is viewed as an $O_{X} G$-supermodule with structure map $\rho_{*}\left(\operatorname{id}_{\mathcal{M}} \otimes \mu^{\#}\right):$ ind $\mathcal{M} \rightarrow \rho_{*}\left(\right.$ ind $\left.M \otimes O_{G}\right)$.

Now take any $\mathcal{O}_{X} G$-supermodule $\mathcal{M}$ with structure map $\eta: \mathcal{M} \rightarrow \rho_{*}\left(\mathcal{M} \otimes \mathscr{O}_{G}\right)$. Embed res $\mathcal{M}$ into an injective quasicoherent $\mathcal{O}_{X}$-supermodule $\mathcal{N}$ (which we can do since $\mathfrak{q} \mathfrak{c o h} \mathfrak{O}_{X}$ has enough injectives; see [Hartshorne 1977, III.3, ex. 6]). Applying the left exact functor ind, we get a monomorphism ind $\circ \operatorname{res}(\mathcal{M}) \rightarrow \operatorname{ind}(\mathcal{N})$, with $\operatorname{ind}(\mathcal{N})$ being injective. It remains to see that $\mathcal{M}$ embeds into ind $\circ \operatorname{res}(\mathcal{M})$; but this is immediate since $\eta: \mathcal{M} \rightarrow$ ind $\circ \operatorname{res}(\mathcal{M})$ is a monomorphism thanks to axiom (4).

(ii) By the proof of (i), any injective object in $\mathfrak{q} \mathfrak{c o h} \mathfrak{O}_{X} G$ embeds into (hence is a summand of) $\rho_{*}\left(M \otimes \mathscr{O}_{G}\right)$ for an injective object $\mathcal{M} \in \mathfrak{q c o h}_{\mathscr{O}_{X}}$. So it suffices to check that $H^{i}\left(X, \rho_{*}\left(M \otimes \mathcal{O}_{G}\right)\right)=0$ for all $i>0$. Note $\rho$ is the composite

$$
X \times G \stackrel{f}{\longrightarrow} X \times G \stackrel{\mathrm{pr}_{X}}{\longrightarrow} X,
$$

where $f$ is the isomorphism defined by $(x, g) \mapsto(x g, g)$. Hence, $\rho$ is an affine morphism, so $\left(R^{i} \rho_{*}\right)\left(M \otimes \mathcal{O}_{G}\right)=0$ for all $i>0$. Hence the Leray spectral sequence degenerates to give $H^{i}\left(X, \rho_{*}\left(M \otimes \mathcal{O}_{G}\right)\right) \simeq H^{i}\left(X \times G, M \otimes \mathcal{O}_{G}\right)$. Now by Serre's 
vanishing theorem and the Kunneth formula, this is isomorphic to $H^{i}(X, \mu) \otimes$ $k[G]$, which is zero for $i>0$ by the injectivity of $\mu$.

Let $\mathcal{M}$ be a quasicoherent $O_{X} G$-supermodule with structure map $\eta$. The space $H^{0}(X, M)$ of global sections has the structure of a $G$-supermodule, with structure map obtained as the composite

$$
H^{0}(X, \mu) \stackrel{\eta}{\longrightarrow} H^{0}\left(X, \rho_{*}\left(\mu \otimes \mathcal{O}_{G}\right)\right) \simeq H^{0}(X, \mu) \otimes k[G] .
$$

It follows from this and Lemma 2.2 that each $H^{i}(X, M)$ carries a canonical $G$ supermodule structure. Indeed, we can compute $H^{i}(X, M)$ using a resolution of $\mu$ by injective $O_{X} G$-supermodules, these being acyclic for $H^{0}(X, ?)$. In other words, we can regard each $H^{i}(X, ?)$ as a functor from $\mathfrak{q c o h}_{\mathbb{O}_{X} G}$ to $\mathfrak{m o d}_{G}$.

Suppose next that $H$ is a closed subgroup of our fixed algebraic supergroup $G$. By a quotient of $G$ by $H$ (" $H \backslash G$ ") we mean here a noetherian superscheme $X$ together with a morphism $\pi: G \rightarrow X$ such that:

(Q1) $\pi$ is constant on the right $H(A)$-cosets $H(A) g$ in $G(A)$ for each commutative superalgebra $A$ and each $g \in G(A)$;

(Q2) given any other morphism $f: G \rightarrow Y$ of superschemes that is constant on the right $H(A)$-cosets in $G(A)$ for each commutative superalgebra $A$, there is a unique $\tilde{f}: X \rightarrow Y$ such that $f=\tilde{f} \circ \pi$;

(Q3) $\pi: G \rightarrow X$ is a faithfully flat, affine morphism.

Assume that $X$ is a quotient of $G$ by $H$. Let $\rho: X \times G \rightarrow X$ be the right action of $G$ on $X$ induced by multiplication in $G$. We have commutative diagrams
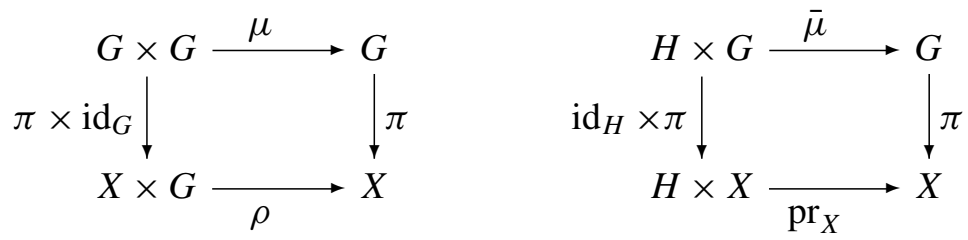

where $\mu$ is the multiplication in $G$ and $\bar{\mu}$ is its restriction to $H \times G$.

The first diagram gives that $\pi_{*} \mathrm{O}_{G}$ is a quasicoherent $\mathrm{O}_{X} G$-supermodule with structure map $\pi_{*} \mu^{\#}: \pi_{*} O_{G} \rightarrow \rho_{*}\left(\pi_{*} O_{G} \otimes O_{G}\right)$. Hence, for any vector superspace $M, M \otimes \pi_{*} \mathcal{O}_{G}$ is also a quasicoherent $O_{X} G$-supermodule with structure map $\operatorname{id}_{M} \otimes \pi_{*} \mu^{\#}$. We will usually denote this by $M_{t r} \otimes \pi_{*} O_{G}$ to indicate that the action on $M$ is trivial. In particular, $k[H]_{t r} \otimes \pi_{*} O_{G}$ is a quasicoherent $O_{X} G$-supermodule in this way. From the second diagram, we get a natural $O_{X} G$-supermodule map $\delta:=\pi_{*} \bar{\mu}^{\#}: \pi_{*} \widehat{O}_{G} \rightarrow k[H]_{t r} \otimes \pi_{*} \hat{O}_{G}$. If $M$ is an $H$-supermodule with structure map $\eta: M \rightarrow M \otimes k[H]$ (see [Brundan and Kleshchev 2003, §2]), we define the "induced" quasicoherent $\mathrm{O}_{X} G$-supermodule $\mathscr{L}(M)$ to be the kernel of the map 
$\partial=\eta \otimes \mathrm{id}_{\pi_{*} O_{G}}-\mathrm{id}_{M} \otimes \delta$ in the following exact sequence of quasicoherent $O_{X} G$ supermodules:

$$
0 \longrightarrow \mathscr{L}(M) \longrightarrow M_{t r} \otimes \pi_{*} \mathrm{O}_{G} \stackrel{\partial}{\longrightarrow} M_{t r} \otimes k[H]_{t r} \otimes \pi_{*} \mathrm{O}_{G} .
$$

If $f: M \rightarrow M^{\prime}$ is a morphism of $H$-supermodules, $\mathscr{L}(f): \mathscr{L}(M) \rightarrow \mathscr{L}\left(M^{\prime}\right)$ can be defined as the restriction of $f \otimes \mathrm{id}_{\pi_{*} O_{G}}$, giving that

$$
\mathscr{L}: \mathfrak{m o d}_{H} \rightarrow \mathfrak{q c o h}_{O_{X} G}
$$

is a functor, where $\mathfrak{m o d}_{H}$ denotes the category of all $H$-supermodules.

Conversely, recall that $e: \operatorname{Spec} k \rightarrow G$ denotes the identity of $G$; we let $\bar{e}$ : Spec $k \rightarrow X$ denote $\pi \circ e$. There is a natural $H$-supermodule structure on $M(\bar{e}):=$ $\mathcal{M}_{\bar{e}} \otimes_{\mathcal{O}_{X, \bar{e}}} k$. In other words, "evaluation at $\bar{e}$ " gives a functor

$$
\mathscr{E}: \mathfrak{q c o h} \mathfrak{O}_{X} G \rightarrow \mathfrak{m o d}_{H}
$$

In terms of the associated action of $G$ on the fibration $\mathbb{V}(\mathcal{M})$, the restriction of the action to $H$ induces a right action of $H$ on the fiber $\mathbb{V}(\mathcal{M})_{\bar{e}}$. There is a natural isomorphism $\mathbb{V}(\mathcal{M})_{\bar{e}}=\operatorname{Spec}_{X}(S(\mathcal{M})) \times_{X} \operatorname{Spec} k \cong \operatorname{Spec} S(\mathcal{M}(\bar{e})$ ) (see [Demazure and Gabriel 1970, I, §2, 3.6] and [Hartshorne 1977, II, ex. 5.16(e)]), so that $H$ acts on the right on $\operatorname{Spec} S(M(\bar{e}))$. Now the structure map on $M(\bar{e})$ is precisely the restriction to $\mathcal{M}(\bar{e})$ of the comorphism of this right action of $H$ on $\operatorname{Spec} S(M(\bar{e}))$.

We are ready state the following fundamental result, for which see [Demazure 1969/1970, p. 249] or [Cline et al. 1983, Theorem (2.7)]. We omit the proof, but note that it depends in an essential way on the property (Q3) of quotients as formulated above.

Theorem 2.3. The functors $\mathscr{L}$ and $\mathscr{E}$ are mutually inverse equivalences. Moreover, for an $H$-supermodule $M, \mathscr{L}(M)$ is locally free of rank equal to the superdimension of the vector superspace $M$.

There are induction and restriction functors

$$
\operatorname{ind}_{H}^{G}: \mathfrak{m o d}_{H} \rightarrow \mathfrak{m o d}_{G}, \quad \operatorname{res}_{H}^{G}: \mathfrak{m o d}_{G} \rightarrow \mathfrak{m o d}_{H}
$$

defined in [Brundan and Kleshchev 2003, §6]. We recall that restriction is exact and ind ${ }_{H}^{G}$ is right adjoint to $\operatorname{res}_{H}^{G}$. Comparing the formula [Brundan and Kleshchev 2003, (6.1)] with (5), it is immediate that there is an isomorphism

$$
\operatorname{ind}_{H}^{G} \simeq H^{0}(X, ?) \circ \mathscr{L}
$$

of functors from $\mathfrak{m o d}_{H}$ to $\mathfrak{m o d}_{G}$. By the theorem, the functor $\mathscr{L}$ is exact and maps injectives to injectives. So, letting $R^{i} \operatorname{ind}_{H}^{G}$ denote the $i$ th right derived functor of induction, we have: 
Corollary 2.4. For each $i \geq 0$, we have

$$
R^{i} \operatorname{ind}_{H}^{G} \simeq H^{i}(X, ?) \circ \mathscr{L} .
$$

The goal in the remainder of the section is to sketch the proof of a result from [Penkov 1988, §2.3] about Euler characteristics. Let $\pi: G \rightarrow X$ be a quotient of $G$ by $H$ as before. We now make the following additional assumptions:

(Q4) the restriction $\pi_{\mathrm{ev}}: G_{\mathrm{ev}} \rightarrow X_{\mathrm{ev}}$ of $\pi$ to the underlying purely even schemes is a quotient of $G_{\mathrm{ev}}$ by $H_{\mathrm{ev}}$;

(Q5) $X$ is locally decomposable.

Write $i: X_{\mathrm{ev}} \rightarrow X$ for the canonical closed immersion. The map $i$ is $G_{\mathrm{ev}^{-}}$ equivariant, so taking direct and inverse images as $\mathrm{O}_{X}$-supermodules give functors

$$
i_{*}: \mathfrak{m o d}_{\bigcup_{X_{\mathrm{ev}}} G_{\mathrm{ev}}} \rightarrow \mathfrak{m o d}_{\mathscr{O}_{X} G_{\mathrm{ev}}}, \quad i^{*}: \mathfrak{m o d}_{\mathscr{O}_{X} G_{\mathrm{ev}}} \rightarrow \mathfrak{m o d}_{\mathscr{O}_{X_{\mathrm{ev}}} G_{\mathrm{ev}}} .
$$

There is a natural restriction functor $\operatorname{res}_{G_{\mathrm{ev}}}^{G}: \mathfrak{q c o h}_{\mathscr{O}_{X} G} \rightarrow \mathfrak{q c o h}_{\mathscr{O}_{X} G_{\mathrm{ev}}}$, and obviously

$$
H^{i}\left(X, \operatorname{res}_{G_{\mathrm{ev}}}^{G} \mu\right) \simeq \operatorname{res}_{G_{\mathrm{ev}}}^{G} H^{i}(X, \mathcal{M})
$$

for any quasicoherent $\mathrm{O}_{X} G$-supermodule $\mathcal{M}$. Finally, by a special case of the above theory, applied to the quotient $\pi_{\mathrm{ev}}: G_{\mathrm{ev}} \rightarrow X_{\mathrm{ev}}$ of $G_{\mathrm{ev}}$ by $H_{\mathrm{ev}}$, we have inverse equivalences

$$
\mathscr{L}_{\mathrm{ev}}: \mathfrak{m} \mathfrak{o d} \mathfrak{d}_{H_{\mathrm{ev}}} \rightarrow \mathfrak{q} \mathfrak{c o h} \mathfrak{C}_{\widehat{O}_{\mathrm{Xv}} G_{\mathrm{ev}}}, \quad \mathscr{E}_{\mathrm{ev}}: \mathfrak{q} \mathfrak{c o h} \mathfrak{O}_{\widehat{X}_{\mathrm{ev}} G_{\mathrm{ev}}} \rightarrow \mathfrak{m o d} \mathfrak{d}_{H_{\mathrm{ev}}}
$$

Lemma 2.5. For any $H$-supermodule $M$, we have

$$
i^{*}\left(\operatorname{res}_{G_{\mathrm{ev}}}^{G} \mathscr{L}(M)\right) \simeq \mathscr{L}_{\mathrm{ev}}\left(\operatorname{res}_{H_{\mathrm{ev}}}^{H} M\right) .
$$

Proof. Let $\mathcal{M}$ be a quasicoherent $\mathrm{O}_{X} G$-supermodule. Then,

$$
\begin{aligned}
\mathbb{V}\left(i^{*} \mathcal{M}\right)_{\bar{e}} & \simeq\left(\mathbb{V}(\mathcal{M}) \times_{X} X_{\mathrm{ev}}\right)_{\bar{e}}=\mathbb{V}(\mathcal{M}) \times_{X} X_{\mathrm{ev}} \times{ }_{X_{\mathrm{ev}}} \operatorname{Spec} k \\
\simeq \mathbb{V}(\mathcal{M}) \times_{X} \operatorname{Spec} k & =\mathbb{V}(\mathcal{M})_{\bar{e}} .
\end{aligned}
$$

Hence, $\mathscr{E}_{\mathrm{ev}}\left(i^{*} \mathcal{M}\right) \simeq \mathscr{E}(\mathcal{M})$ as $H_{\mathrm{ev}}$-supermodules. The lemma follows on applying the functor $\mathscr{L}_{\mathrm{ev}}$ to both sides.

Suppose that $\mathcal{M}$ is a quasicoherent $\mathcal{O}_{X} G$-supermodule. The canonical filtration (2) of $\mathcal{M}$ is a filtration as an $\mathscr{O}_{X} G_{\mathrm{ev}}$-supermodule (for instance, $\mathscr{F}_{X} \mathcal{M}$ is the kernel of the canonical map $\mathcal{M} \rightarrow i_{*} i^{*} \mathcal{M}$ ). The factors are $\mathcal{O}_{X_{\mathrm{ev}}} G_{\mathrm{ev}}$-supermodules.

Lemma 2.6. There is an isomorphism

$$
\mathscr{F}_{X} / \mathscr{F}_{X}^{2} \simeq \mathscr{L}_{\mathrm{ev}}\left((\text { Lie } G / \text { Lie } H)_{\frac{1}{1}}^{*}\right)
$$

as $\mathcal{O}_{X_{\mathrm{ev}}} G_{\mathrm{ev}}$-supermodules. 
Proof. The sheaf $\Omega_{X}$ of (super) Kähler differentials on $X$ (over $k$ ) has a natural $G$ structure, and the closed immersion $i: X_{\mathrm{ev}} \rightarrow X$ induces a standard exact sequence

$$
\mathscr{F}_{X} / \mathscr{F}_{X}^{2} \stackrel{\delta}{\longrightarrow} \Omega_{X} \otimes_{0_{X}} \mathrm{O}_{X_{\mathrm{ev}}} \longrightarrow \Omega_{X_{\mathrm{ev}}} \longrightarrow 0
$$

of ${ }^{O} X_{\mathrm{ev}} G_{\mathrm{ev}}$-supermodules (see [Hartshorne 1977, II.8.12], with appropriate modifications in the super case). Using the assumption that $X$ is locally decomposable, one checks by reducing to the case that $X$ is affine and decomposable that the map $\delta$ is a monomorphism. Now we note that $\mathscr{E}\left(\Omega_{X}\right) \simeq(\operatorname{Lie}(G) / \operatorname{Lie}(H))^{*}$ and $\mathscr{E}_{\mathrm{ev}}\left(\Omega_{X_{\mathrm{ev}}}\right) \simeq\left(\operatorname{Lie}\left(G_{\mathrm{ev}}\right) / \operatorname{Lie}\left(H_{\mathrm{ev}}\right)\right)^{*}$. Applying the equivalence of categories $\mathscr{E}_{\mathrm{ev}}$ and Lemma 2.5, we obtain an exact sequence

$$
0 \longrightarrow \mathscr{E}_{\mathrm{ev}}\left(\mathscr{F}_{X} / \mathscr{F}_{X}^{2}\right) \longrightarrow(\operatorname{Lie}(G) / \operatorname{Lie}(H))^{*} \longrightarrow\left(\operatorname{Lie}\left(G_{\mathrm{ev}}\right) / \operatorname{Lie}\left(H_{\mathrm{ev}}\right)\right)^{*} \longrightarrow 0
$$

of $H_{\mathrm{ev}}$-supermodules. Since $\operatorname{Lie}\left(G_{\mathrm{ev}}\right)=\operatorname{Lie}(G)_{\overline{0}}$ and similarly for $H$, we deduce that

$$
\mathscr{E}_{\mathrm{ev}}\left(\mathscr{F}_{X} / \mathscr{F}_{X}^{2}\right) \simeq(\operatorname{Lie}(G) / \operatorname{Lie}(H))_{1}^{*}
$$

and the lemma follows.

Theorem 2.7. Let $M$ be an $H$-supermodule. Then, the factors in the canonical filtration of $\mathscr{L}(M)$ satisfy

$$
\mathscr{F}_{X}^{i} \mathscr{L}(M) / \mathscr{F}_{X}^{i+1} \mathscr{L}(M) \simeq \mathscr{L}_{\mathrm{ev}}\left(S^{i}\left[(\operatorname{Lie} G / \text { Lie } H)_{1}^{*}\right] \otimes \operatorname{res}_{H_{e v}}^{H} M\right)
$$

as $0_{X_{\mathrm{ev}}} G_{\mathrm{ev}}$-supermodules.

Proof. Note $\mathscr{L}(M)$ is locally free by Theorem 2.3. So we obtain from Lemma 2.1 an isomorphism

$$
\mathscr{F}_{X}^{i} \mathscr{L}(M) / \mathscr{F}_{X}^{i+1} \mathscr{L}(M) \simeq S^{i}\left(\mathscr{F}_{X} / \mathscr{F}_{X}^{2}\right) \otimes_{\mathcal{O}_{\mathrm{ev}}} \mathcal{M} / \mathscr{F}_{X} M .
$$

This is actually an isomorphism as $0_{X_{\mathrm{ev}}} G_{\mathrm{ev}}$-supermodules. So the theorem follows using Lemmas 2.5 and 2.6. (We have noted that the usual operations of tensor algebra commute with the functor $\mathscr{L}_{\mathrm{ev}}$ ).

For the final corollary, we make one further assumption:

(Q6) $X_{\text {ev }}$ is projective.

Corollary 2.8. For any finite-dimensional $H$-supermodule $M$, we have

$$
\sum_{i \geq 0}(-1)^{i}\left(\operatorname{res}_{G_{\mathrm{ev}}}^{G} R^{i} \operatorname{ind}_{H}^{G} M\right)=\sum_{i \geq 0}(-1)^{i}\left(R^{i} \operatorname{ind}_{H_{\mathrm{ev}}}^{G_{\mathrm{ev}}}\left(S\left((\text { Lie } G / \text { Lie } H)_{1}^{*}\right) \otimes M\right)\right),
$$

where the equality is written in the Grothendieck group of finite-dimensional $G_{\mathrm{ev}^{-}}$ supermodules. 
Proof. By Theorem 2.3, Corollary 2.4 and Serre's finiteness theorem, the functor $R^{i}$ ind $_{H}^{G}$ sends finite-dimensional $H$-supermodules to finite dimensional $G$ supermodules, and similarly for $R^{i}$ ind $_{H_{\mathrm{ev}}}^{G_{\mathrm{ev}}}$. So all modules appearing in the formula are finite-dimensional. Moreover, only finitely many terms in either summation are nonzero by Grothendieck vanishing. So the formula at least makes sense. Now combine (6), Corollary 2.4, Theorem 2.7 and additivity of Euler characteristics.

\section{Flag superschemes of type $Q(n)$}

Now we are ready to introduce the supergroup $G=Q(n)$ into the picture. Recall from [Brundan and Kleshchev 2003, §3] that $G$ is the functor from the category of commutative superalgebras to the category of groups defined on a superalgebra $A$ so that $G(A)$ is the group of all invertible $2 n \times 2 n$ matrices of the form

$$
g=\left(\begin{array}{c|c}
S & S^{\prime} \\
\hline-S^{\prime} & S
\end{array}\right)
$$

where $S$ is an $n \times n$ matrix with entries in $A_{\overline{0}}$ and $S^{\prime}$ is an $n \times n$ matrix with entries in $A_{\overline{1}}$. The underlying even group $G_{\mathrm{ev}}$ is isomorphic to $\mathrm{GL}(n)$.

We also need the closed subgroup $H$ of $G$ defined on a commutative superalgebra $A$ so that $H(A)$ consists of all matrices of the form (7) with $S, S^{\prime}$ being diagonal matrices, and the standard Borel subgroup $B$ of $G$ defined so that $B(A)$ consists of matrices with $S, S^{\prime}$ being lower triangular. Let $T=H_{\mathrm{ev}}$ be the standard $n$-dimensional maximal torus of $G_{\mathrm{ev}}$. Let $X(T)$ be the character group of $T$, the free abelian group on generators $\varepsilon_{1}, \ldots, \varepsilon_{n}$ where $\varepsilon_{i}: T \rightarrow \mathbb{G}_{m}$ picks out the $i$ th diagonal entry. The root system associated to $G_{\mathrm{ev}}$ is denoted $R=R^{+} \cup\left(-R^{+}\right)$, where $R^{+}=\left\{\varepsilon_{i}-\varepsilon_{j} \mid 1 \leq i<j \leq n\right\} \subset X(T)$. We partially order $X(T)$ by the usual dominance order, so $\lambda \leq \mu$ if and only if $\mu-\lambda$ is a sum of positive roots.

For $\lambda=\sum_{i=1}^{n} \lambda_{i} \varepsilon_{i} \in X(T)$, we write $x^{\lambda}=x_{1}^{\lambda_{1}} \ldots x_{n}^{\lambda_{n}} \in \mathbb{Z}\left[x_{1}^{ \pm 1}, \ldots, x_{n}^{ \pm 1}\right]$. The Weyl group $W \cong S_{n}$ associated to $G_{\text {ev }}$ acts naturally on $X(T)$ hence on $\mathbb{Z}\left[x_{1}^{ \pm 1}, \ldots, x_{n}^{ \pm 1}\right]$. The character

$$
\operatorname{ch} M:=\sum_{\lambda \in X(T)}\left(\operatorname{dim} M_{\lambda}\right) x^{\lambda}
$$

of a finite-dimensional $G$-supermodule $M$ is naturally $W$-invariant, so is an element of the ring $\mathbb{Z}\left[x_{1}^{ \pm 1}, \ldots, x_{n}^{ \pm 1}\right]^{W}$ of symmetric functions.

The irreducible $G$-supermodules are classified in [Brundan and Kleshchev 2003, Theorem 6.11] by their highest weights. For every $\lambda=\sum_{i=1}^{n} \lambda_{i} \varepsilon_{i} \in X(T)$, there is by [Brundan and Kleshchev 2003, Lemma 6.4] a unique irreducible $H$-supermodule denoted $u(\lambda)$ with character $2^{\left\lfloor\left(h_{p^{\prime}}(\lambda)+1\right) / 2\right\rfloor} x^{\lambda}$, where $h_{p^{\prime}}(\lambda)$ denotes the number of $i=1, \ldots, n$ for which $p \nmid \lambda_{i}$. Let $X_{p}^{+}(T)$ denote the set of all $\lambda=$ 
$\sum_{i=1}^{n} \lambda_{i} \varepsilon_{i} \in X(T)$ such that $\lambda_{1} \geq \cdots \geq \lambda_{n}$, and moreover $\lambda_{i}=\lambda_{i+1}$ implies $p \mid \lambda_{i}$ for each $i=1, \ldots, n-1$. Then, according to [Brundan and Kleshchev 2003, Theorem 6.11], the induced module

$$
H^{0}(\lambda):=\operatorname{ind}_{B}^{G} \mathfrak{u}(\lambda)
$$

is nonzero if and only if $\lambda \in X_{p}^{+}(T)$, and in that case $H^{0}(\lambda)$ has a unique irreducible submodule denoted $L(\lambda)$. The $\left\{L(\lambda) \mid \lambda \in X_{p}^{+}(T)\right\}$ form a complete set of pairwise nonisomorphic irreducible $G$-supermodules. Also by [Brundan and Kleshchev 2003, Lemma 6.10], $L(\lambda)_{\lambda} \simeq \mathfrak{u}(\lambda)$ and the lowest weight of $L(\lambda)$ is $w_{0} \lambda$, where $w_{0}$ is the longest element of $W$.

Next we introduce the standard Levi subgroups of $G$. Just as for GL $(n)$, these can be parametrized by compositions of $n$, i.e., tuples $\gamma=\left(\gamma_{1}, \ldots, \gamma_{s}\right)$ of positive integers summing to $n$. Given such a $\gamma$, the standard Levi subgroup

$$
G_{\gamma} \cong Q\left(\gamma_{1}\right) \times \cdots \times Q\left(\gamma_{s}\right)
$$

of $G$ is defined on a commutative superalgebra $A$ so that $G_{\gamma}(A)$ is the subgroup of $G(A)$ consisting of all elements of the form (7) with $S, S^{\prime}$ being block diagonal matrices, block sizes $\gamma_{1}, \ldots, \gamma_{s}$ down the diagonal. The standard parabolic subgroup $P_{\gamma}$ with Levi factor $G_{\gamma}$ is defined similarly, so $P_{\gamma}(A)$ consists of all elements of the form (7) with $S, S^{\prime}$ being lower triangular block matrices, block sizes $\gamma_{1}, \ldots, \gamma_{s}$ down the diagonal. Also let $U_{\gamma}^{+}$denote the unipotent radical of the opposite parabolic subgroup to $P_{\gamma}$. Thus, $U_{\gamma}^{+}(A)$ consists of all matrices of the form (7) with $S, S^{\prime}$ being upper unitriangular block matrices, block sizes $\gamma_{1}, \ldots, \gamma_{s}$ down the diagonal. The root system associated to $G_{\gamma}$ will be denoted $R_{\gamma} \subseteq R$, and its Weyl group is $W_{\gamma} \leq W$, with longest element denoted $w_{\gamma}$.

Lemma 3.1. Let $\lambda \in X_{p}^{+}(T)$ and $\gamma$ be a composition of $n$ satisfying $w_{\gamma} \lambda=\lambda$. Then, $\operatorname{ind}_{B}^{P_{\gamma}} \mathfrak{u}(\lambda) \simeq \mathfrak{u}(\lambda)$, i.e., the $H$-action on $\mathfrak{u}(\lambda)$ extends uniquely to a $P_{\gamma}$-action.

Proof. As in [Brundan and Kleshchev 2003, Lemma 6.10(i)], the lowest weight of $\operatorname{ind}_{B}^{P_{\gamma}} \mathfrak{u}(\lambda)$ is $w_{\gamma} \lambda=\lambda$.

We need the parabolic analogue of the big cell. For $1 \leq i, j \leq n$, let $s_{i, j}$ resp. $s_{i, j}^{\prime}$ be the coordinate function picking out the $i j$-entry of the matrix $S$ resp. $S^{\prime}$ of $g \in G(A)$ written in the form (7). As in [Brundan and Kleshchev 2003, §3], the coordinate ring $k[G]$ is the free commutative superalgebra on even generators $s_{i, j}$ and odd generators $s_{i, j}^{\prime}$ localized at det $:=\operatorname{det}\left(\left(s_{i, j}\right)_{1 \leq i, j \leq n}\right)$. Instead, for $1 \leq m \leq n$, define $\operatorname{det}_{m}$ to be the determinant of the $m \times m$ matrix $\left(s_{i, j}\right)_{1 \leq i, j \leq m}$. Given a composition $\gamma=\left(\gamma_{1}, \ldots, \gamma_{s}\right)$ of $n$, set

$$
\operatorname{det}_{\gamma}=\operatorname{det}_{\gamma_{1}} \operatorname{det}_{\gamma_{1}+\gamma_{2}} \ldots \operatorname{det}_{\gamma_{1}+\gamma_{2}+\cdots+\gamma_{s}} .
$$


We will denote the principal open subset of $G$ defined by det $\operatorname{de}_{\gamma}$ by $\Omega_{\gamma}$; so $\Omega_{\gamma}$ is an affine superscheme with coordinate ring $k\left[\Omega_{\gamma}\right]$ being the localization of $k[G]$ at $\operatorname{det}_{\gamma}$. Proceeding by induction on the number $s$ of blocks, one shows as in [Brundan and Kleshchev 2003, Theorem 3.5] that:

Lemma 3.2. Multiplication defines an isomorphism of affine superschemes between $P_{\gamma} \times U_{\gamma}^{+}$and $\Omega_{\gamma}$.

Let $V$ be the natural $G$-supermodule. Thus, $V$ is the vector superspace on basis $v_{1}, \ldots, v_{n}, v_{1}^{\prime}, \ldots, v_{n}^{\prime}$, where $v_{i}$ is even and $v_{i}^{\prime}$ is odd. For a superalgebra $A$, we identify elements of $V \otimes A$ with column vectors

$$
\sum_{i=1}^{n}\left(v_{i} \otimes a_{i}+v_{i}^{\prime} \otimes a_{i}^{\prime}\right) \longleftrightarrow\left(\begin{array}{c}
a_{1} \\
\vdots \\
a_{n} \\
a_{1}^{\prime} \\
\vdots \\
a_{n}^{\prime}
\end{array}\right) .
$$

Then, the action of $G(A)$ on $V \otimes A$ defining the supermodule structure is the obvious action on column vectors by left multiplication. Moreover, the map $J$ : $V \rightarrow V$ taking each $v_{i}$ to $v_{i}^{\prime}$ and each $v_{i}^{\prime}$ to $-v_{i}$ is an odd automorphism of $V$ as a $G$-supermodule.

Instead, let $\widetilde{V}$ denote the affine superscheme defined on a superalgebra $A$ by $\widetilde{V}(A):=\operatorname{Hom}_{k}(V, A)$ and on a morphism $\theta: A \rightarrow B$ of superalgebras by $\widetilde{V}(\theta)$ : $\widetilde{V}(A) \rightarrow \widetilde{V}(B), f \mapsto \theta \circ f$. Note $\widetilde{V}(A)$ is a free left $A$-supermodule of rank $n \mid n$, with action defined by $(a \cdot f)(v)=a(f(v))$. Indeed, we can identify functions in $\widetilde{V}(A)$ with row vectors so that

$$
f \longleftrightarrow\left(f\left(v_{1}\right), \ldots, f\left(v_{n}\right), f\left(v_{1}^{\prime}\right), \ldots, f\left(v_{n}^{\prime}\right)\right) .
$$

Now right multiplication defines a right action of $G(A)$ on $\tilde{V}(A)$ by $A$-supermodule automorphisms for each $A$. Hence we have a right action $\rho: \tilde{V} \times G \rightarrow \tilde{V}$ of $G$ on the superscheme $\tilde{V}$. There is a $G$-equivariant morphism $\tilde{J}: \widetilde{V} \rightarrow \widetilde{V}$, defined by $\tilde{J}(f)(v)=(-1)^{\bar{f}} f(J(v))$ for each $v \in V, f \in \widetilde{V}(A)$ and each superalgebra $A$.

At last we can introduce the flag superscheme $X_{\gamma}$ corresponding to a composition $\gamma=\left(\gamma_{1}, \ldots, \gamma_{s}\right)$ of $n$. Let $d_{i}=\gamma_{1}+\cdots+\gamma_{i}$ for short. Given a superalgebra $A$, a $J$-invariant $\gamma$-flag in $\widetilde{V}(A)$ means a chain $\left(f_{1} \subseteq \cdots \subseteq f_{s}\right)$ of $J$-invariant direct summands of the free $A$-supermodule $\tilde{V}(A)$, where each $f_{i}$ has rank $d_{i} \mid d_{i}$ as a projective $A$-supermodule. Let $X_{\gamma}$ denote the functor mapping a superalgebra $A$ to the set $X_{\gamma}(A)$ of all such $J$-invariant $\gamma$-flags in $\widetilde{V}(A)$. For a morphism $\theta: A \rightarrow B$, $X_{\gamma}(\theta): X_{\gamma}(A) \rightarrow X_{\gamma}(B)$ is the map induced by composing with $\theta$. 
The right action of $G$ on $\widetilde{V}$ induces a right action $\rho: X_{\gamma} \times G \rightarrow X_{\gamma}$ of $G$ on $X_{\gamma}$. Let $f_{\gamma}=\left(f_{1} \subseteq \cdots \subseteq f_{s}\right) \in X_{\gamma}(k)$ denote the standard $\gamma$-flag, where $f_{i}$ is the direct summand of $\tilde{V}(k)=\operatorname{Hom}_{k}(V, k)$ consisting of all functions annihilating $v_{d_{i}+1}, \ldots, v_{n}, v_{d_{i}+1}^{\prime}, \ldots, v_{n}^{\prime}$. Note that $\operatorname{stab}_{G} f_{\gamma}=P_{\gamma}$, i.e. $P_{\gamma}(A)$ is the stabilizer in $G(A)$ of the canonical image of $f_{\gamma}$ in $X_{\gamma}(A)$ for each superalgebra $A$. We get a corresponding orbit map

$$
\pi_{\gamma}: G \rightarrow X_{\gamma}
$$

defined for each superalgebra $A$ and $g \in G(A)$ by $\pi_{\gamma}(g):=f_{\gamma} \cdot g$, which is constant on right $P_{\gamma}(A)$-cosets in $G(A)$ for each $A$. In fact, according to a result of Manin, $\pi_{\gamma}: G \rightarrow X_{\gamma}$ is the quotient of $G$ by the parabolic subgroup $P_{\gamma}$ :

Theorem 3.3. $\pi_{\gamma}: G \rightarrow X_{\gamma}$ satisfies the properties $(Q 1)-(Q 6)$ from the previous section.

The properties (Q4) and (Q6) hold because the underlying even scheme $X_{\gamma, \text { ev }}$ is precisely the usual flag variety of $\operatorname{GL}(n)$. For the remaining properties, the main step is to show that $X_{\gamma}$ really is a superscheme, for which we refer to [Manin 1997, ch. $4, \S 3 ; 1991, \S 3.1]$. One can also give a proof directly in the functorial language that $X_{\gamma}$ is a superscheme along the lines of the arguments in [Demazure and Gabriel $1970, I, \S 1,3.13]$, by constructing an affine open cover and checking directly that $X_{\gamma}$ is local in the sense of [Demazure and Gabriel 1970, I, §1, 3.11]. We just describe the construction of the affine open cover here; all the other properties claimed follow easily given this.

Let $e_{\gamma}=\left(e_{1} \subseteq \cdots \subseteq e_{s}\right)$ be the $J$-invariant $\gamma$-flag in the vector superspace $V$ with $e_{i}=\operatorname{span}\left\{v_{1}, \ldots, v_{d_{i}}, v_{1}^{\prime}, \ldots, v_{d_{i}}^{\prime}\right\}$. Let $U_{1}$ denote the subfunctor of $X_{\gamma}$ defined by

$$
U_{1}(A)=\left\{\begin{array}{l|l}
\left(f_{1} \subseteq \cdots \subseteq f_{s}\right) \in X_{\gamma}(A) & \begin{array}{l}
f_{i} \text { is a complement to } \\
\operatorname{ann}_{\tilde{V}(A)}\left(e_{i}\right) \text { in } \widetilde{V}(A)
\end{array}
\end{array}\right\}
$$

for each superalgebra $A$. Then, $U_{1}$ is an open subfunctor of $X_{\gamma}, \pi_{\gamma}^{-1}\left(U_{1}\right)$ is the big cell $P_{\gamma} U_{\gamma}^{+}=\Omega_{\gamma} \subset G$ (see Lemma 3.2), and the restriction of $\pi_{\gamma}$ to $U_{\gamma}^{+}$gives an isomorphism $U_{\gamma}^{+} \stackrel{\sim}{\rightarrow} U_{1}$. It follows that for any $w \in W$, the translate $U_{w}:=U_{1} \cdot w^{-1}$ is isomorphic to $U_{\gamma}^{+}$, hence it is a decomposable open affine subfunctor of $X_{\gamma}$. For each $A$ that is a field, the $\left\{U_{w}(A) \mid w \in W\right\}$ cover $X_{\gamma}(A)$. Moreover, $U_{w}=U_{w^{\prime}}$ if and only if $w W_{\gamma}=w^{\prime} W_{\gamma}$. Hence

$$
\left\{U_{w} \mid w \in W^{\gamma}\right\}
$$

is the desired affine open cover of $X_{\gamma}$, where $W^{\gamma}$ denotes the set of minimal length $W / W_{\gamma}$-coset representatives. 


\section{Euler characteristics and Schur's $P$-functions}

Now we combine the results of the previous two sections. Continue with the notation of $\S 3$. Let $\lambda=\sum_{i=1}^{n} \lambda_{i} \varepsilon_{i} \in X_{p}^{+}(T)$. Gathering together equal parts, we can write $\left(\lambda_{1}, \ldots, \lambda_{n}\right)=\left(a_{1}^{\gamma_{1}}, a_{2}^{\gamma_{2}}, \ldots, a_{s}^{\gamma_{s}}\right)$ with $a_{1}>a_{2}>\cdots>a_{s}$ and $\sum_{i=1}^{s} \gamma_{i}=n$. We thus obtain from $\lambda$ a composition $\gamma(\lambda):=\left(\gamma_{1}, \ldots, \gamma_{s}\right)$ of $n$. Schur's P-function $p_{\lambda}$ is defined by:

$$
p_{\lambda}=\sum_{w \in W^{\gamma(\lambda)}} w\left(x^{\lambda} \prod_{\substack{1 \leq i<j \leq n \\ \lambda_{i}>\lambda_{j}}} \frac{x_{i}+x_{j}}{x_{i}-x_{j}}\right) .
$$

This is the definition from [Macdonald 1995, III (2.2)] with $t$ there equal to -1 ; compare [Macdonald 1995, III.8]. (Actually, Macdonald only describes the case when all $\lambda_{i} \geq 0$, but everything easily extends to $\lambda_{i} \in \mathbb{Z}$.) For any $\lambda \in X(T)$, let $a_{\lambda}=\sum_{w \in W} \operatorname{sgn}(w) w x^{\lambda}$. Writing $\rho=\sum_{i=1}^{n}(n-i) \varepsilon_{i} \in X^{+}(T), a_{\rho}$ is the Weyl denominator and equals $\prod_{1 \leq i<j \leq n}\left(x_{i}-x_{j}\right)$ by [Macdonald 1995, I.3]. The classical Schur function $s_{\lambda}$ can then be defined for arbitrary $\lambda \in X(T)$ by $s_{\lambda}:=a_{\lambda+\rho} / a_{\rho}$.

Lemma 4.1. For $\lambda \in X_{p}^{+}(T)$,

(i) $p_{\lambda}=\sum_{w \in W} \operatorname{sgn}(w) w\left(x^{\lambda+\rho} \prod_{\substack{1 \leq i<j \leq n \\ \lambda_{i}>\lambda_{j}}}\left(1+x_{i}^{-1} x_{j}\right)\right) / \prod_{1 \leq i<j \leq n}\left(x_{i}-x_{j}\right)$;

(ii) $p_{\lambda}=\sum_{S \subseteq R^{+}-R_{\gamma(\lambda)}^{+}} s_{\lambda-\sum S}$

Proof. (i) The given expression equals

$$
\sum_{w \in W} w\left(x^{\lambda+\rho} \frac{\prod_{\lambda_{i}>\lambda_{j}}\left(1+x_{i}^{-1} x_{j}\right)}{\prod_{i<j}\left(x_{i}-x_{j}\right)}\right)=\sum_{w \in W} w\left(x^{\lambda} \prod_{\substack{1 \leq i<j \leq n \\ \lambda_{i}>\lambda_{j}}} \frac{x_{i}+x_{j}}{x_{i}-x_{j}} \prod_{\substack{1 \leq i<j \leq n \\ \lambda_{i}=\lambda_{j}}} \frac{x_{i}}{x_{i}-x_{j}}\right) .
$$

Now [Macdonald 1995, III(1.4)] with $t=0$ shows that

$$
\sum_{w \in W} w\left(\prod_{i<j} \frac{x_{i}}{x_{i}-x_{j}}\right)=1 \text {. }
$$

The conclusion now follows on observing that $w \in W_{\gamma(\lambda)}$ fixes

$$
x^{\lambda} \prod_{\lambda_{i}>\lambda_{j}} \frac{\left(x_{i}+x_{j}\right)}{\left(x_{i}-x_{j}\right)} .
$$


(ii) We note that

$$
\prod_{\lambda_{i}>\lambda_{j}}\left(1+x_{i}^{-1} x_{j}\right)=\sum_{S \subseteq R^{+}-R_{\gamma(\lambda)}^{+}} x^{-\sum S} .
$$

Now (ii) follows immediately using (i).

Schur's $P$-functions arise naturally for us as certain Euler characteristics. Suppose that $\lambda \in X_{p}^{+}(T)$, and define $\gamma=\gamma(\lambda)$ as above. By Lemma 3.1, we can view $\mathfrak{u}(\lambda)$ as a $P_{\gamma}$-supermodule. For any $i \geq 0$, define

$$
H^{i}(\lambda):=R^{i} \operatorname{ind}_{P_{\gamma}}^{G} \mathfrak{u}(\lambda) .
$$

We note that $\operatorname{ind}_{B}^{G} \mathfrak{u}(\lambda) \simeq \operatorname{ind}_{P_{\gamma}}^{G}\left(\operatorname{ind}_{B}^{P_{\gamma}} \mathfrak{u}(\lambda)\right)=\operatorname{ind}_{P_{\gamma}}^{G} \mathfrak{u}(\lambda)$ by transitivity of induction, i.e. the new definition of $H^{0}(\lambda)$ agrees with the old one from (8).

By Theorem 3.3, the flag superscheme $X_{\gamma}$ is the quotient of $G$ by $P_{\gamma}$. So we also have

$$
H^{i}(\lambda)=H^{i}\left(X_{\gamma}, \mathscr{L}(\mathfrak{u}(\lambda))\right)
$$

invoking Corollary 2.4. Moreover, as in the proof of Corollary 2.8, Grothendieck's vanishing theorem and Serre's finiteness theorem imply:

Lemma 4.2. Each $H^{i}(\lambda)$ is finite-dimensional, and is 0 for $i>\operatorname{dim} X_{\gamma(\lambda)}$.

So we obtain a well-defined element of the character group of $G$ :

$$
E_{\lambda}:=\sum_{i \geq 0}(-1)^{i} \operatorname{ch} H^{i}(\lambda) \in \mathbb{Z}\left[x_{1}^{ \pm 1}, \ldots, x_{n}^{ \pm n}\right]^{W} .
$$

We can compute these explicitly using Corollary 2.8:

Theorem 4.3. For $\lambda \in X_{p}^{+}(T), E_{\lambda}=2^{\left\lfloor\left(h_{p^{\prime}}(\lambda)+1\right) / 2\right\rfloor} p_{\lambda}$.

Proof. Let $\gamma=\gamma(\lambda), P=P_{\gamma}$. For $G=Q(n)$, it is obvious that

$$
(\operatorname{Lie}(G) / \operatorname{Lie}(P))_{1}^{*} \simeq \Pi\left(\operatorname{Lie}\left(G_{\mathrm{ev}}\right) / \operatorname{Lie}\left(P_{\mathrm{ev}}\right)\right)^{*}
$$

as a $G_{\mathrm{ev}}$-supermodule. So Corollary 2.8 tells us that

$$
\sum_{i \geq 0}(-1)^{i} \operatorname{ch} H^{i}(\lambda)=\sum_{i \geq 0}(-1)^{i} \operatorname{ch} R^{i} \operatorname{ind}_{P_{\mathrm{ev}}}^{G_{\mathrm{ev}}}\left(\bigwedge\left(\operatorname{Lie}\left(G_{\mathrm{ev}}\right) / \operatorname{Lie}\left(P_{\mathrm{ev}}\right)\right)^{*} \otimes \mathfrak{u}(\lambda)\right) .
$$

We note that for any $P_{\mathrm{ev}}$-module $M, R^{i} \operatorname{ind}_{P_{\mathrm{ev}}}^{G_{\mathrm{ev}}} M \cong R^{i} \operatorname{ind}_{B_{\mathrm{ev}}}^{G_{\mathrm{ev}}} M$ by [Jantzen 1987, II.4.11] and the tensor identity. Since Euler characteristic is additive on short exact sequences, it therefore suffices to determine the composition factors of the $B_{\mathrm{ev}^{-}}$ module $\bigwedge\left(\operatorname{Lie}\left(G_{\mathrm{ev}}\right) / \operatorname{Lie}\left(P_{\mathrm{ev}}\right)\right)^{*} \otimes \mathfrak{u}(\lambda)$ : its character is

$$
\operatorname{dim} u(\lambda) \sum_{S \subseteq R^{+}-R_{\gamma}^{+}} x^{\lambda-\sum S} .
$$


By Weyl's character formula [Jantzen 1987, II.5.10], we have

$$
\sum_{i \geq 0} \operatorname{ch} R^{i} \operatorname{ind}_{B_{\mathrm{ev}}}^{G_{\mathrm{ev}}} k_{\mu}=s_{\mu}
$$

So

$$
\sum_{i \geq 0} \operatorname{ch} H^{i}(\lambda)=\operatorname{dim} u(\lambda) \sum_{S \subseteq R^{+}-R_{\gamma}^{+}} s_{\lambda-\sum s} .
$$

Now we are done using Lemma 4.1(ii), recalling finally that

$$
\operatorname{dim} \mathfrak{u}(\lambda)=2^{\left\lfloor\left(h_{p^{\prime}}(\lambda)+1\right) / 2\right\rfloor} .
$$

For $\lambda \in X_{p}^{+}(T)$, set $L_{\lambda}:=\operatorname{ch} L(\lambda)$. The sets

$$
\left\{L_{\lambda} \mid \lambda \in X_{p}^{+}(T)\right\}
$$

form a $\mathbb{Z}$-basis for the character group of $G$. So we can write

$$
E_{\lambda}=\sum_{\mu \in X_{p}^{+}(T)} d_{\lambda, \mu} L_{\mu}
$$

for $d_{\lambda, \mu} \in \mathbb{Z}$. The resulting integer matrix $D=\left(d_{\lambda, \mu}\right)_{\lambda, \mu \in X_{p}^{+}(T)}$ is the decomposition matrix of $G$. By Theorem 4.3, $E_{\lambda}$ equals $x^{\lambda}+(*)$ where $(*)$ is a linear combination of $x^{\mu}$ for $\mu<\lambda$, and similarly $L_{\lambda}$ has this form. So we have $d_{\lambda, \lambda}=1$ and $d_{\lambda, \mu}=0$ if $\mu \not \lambda$. Hence, $D$ is a unitriangular matrix if rows and columns are ordered in some way refining dominance, and in particular

$$
\left\{E_{\lambda} \mid \lambda \in X_{p}^{+}(T)\right\}
$$

gives us another natural basis for the character group of $G$. If $p=0$, the decomposition matrices are known explicitly, see [Brundan 2004]. For some examples of decomposition matrices in positive characteristic, see the tables at the end of the article.

We record here one other consequence of Theorem 2.7, giving complete information about the characters of all $R^{i} \operatorname{ind}_{B}^{G} \mathfrak{u}(\lambda)$ in the case $G=Q(2)$ :

Lemma 4.4. Suppose that $G=Q(2)$ and $\lambda \in X(T)$.

(i) For $\lambda \in X_{p}^{+}(T)$, $\operatorname{ch~ind~}_{B}^{G} \mathfrak{u}(\lambda)=2^{\left\lfloor\left(h_{p^{\prime}}(\lambda)+1\right) / 2\right\rfloor}\left(x^{\lambda}+2 x^{\lambda-\alpha}+\cdots+2 x^{w_{0} \lambda+\alpha}+x^{w_{0} \lambda}\right)$.

(ii) For $\lambda \notin X_{p}^{+}(T)$, ch $\operatorname{ind}_{B}^{G} \mathfrak{u}(\lambda)=0$.

(iii) $\operatorname{ch} R^{1} \operatorname{ind}_{B}^{G} \mathfrak{u}(\lambda)=\operatorname{ch} \operatorname{ind}_{B}^{G} \mathfrak{u}\left(w_{0} \lambda\right)$.

In particular, $\operatorname{ind}_{B}^{G} k \simeq k, R^{1} \operatorname{ind}_{B}^{G} k \simeq \Pi k$. 
Proof. Let $X$ denote the quotient of $G$ by $B$. By Theorem 2.7, the canonical filtration gives rise to a short exact sequence of quasicoherent ${ }^{\circ}{ }_{X} G_{\mathrm{ev}}$-supermodules

$$
0 \longrightarrow \mathscr{L}_{\mathrm{ev}}\left(\Pi k_{-\alpha} \otimes \mathfrak{u}(\lambda)\right) \longrightarrow \mathscr{L}(\mathfrak{u}(\lambda)) \longrightarrow \mathscr{L}_{\mathrm{ev}}(\mathfrak{u}(\lambda)) \longrightarrow 0,
$$

where $\alpha=\varepsilon_{1}-\varepsilon_{2}$. Also we already know from the classification [Brundan and Kleshchev 2003, Theorem 6.11] that $\operatorname{ind}_{B}^{G} \mathfrak{u}(\lambda)=0$ unless $\lambda$ is $p$-strict and by Lemma 3.1 that $\operatorname{ind}_{B}^{G} \mathfrak{u}(\lambda) \simeq \mathfrak{u}(\lambda)$ in case $\lambda_{1}=\lambda_{2}$ with $p \mid \lambda_{i}$. The lemma follows on combining these facts, the long exact sequence arising from (11) and the known cohomology of equivariant line bundles on $\mathbb{P}^{1}$.

\section{Serre duality and some examples}

We now give a few simple examples. To do this, we need to make use in addition of Serre duality for the flag superschemes $X_{\gamma}$. We could not find any satisfactory reference for this, but see at least the discussion in [Voronov et al. 1988, §2]. Let us state the theorem:

Theorem 5.1. Let $\gamma$ be a composition of $n$ and $N=\left|R_{\gamma}^{+}\right|$. There is a natural isomorphism

$$
\left(R^{i} \operatorname{ind}_{P_{\gamma}}^{G} M\right)^{*} \cong R^{N-i} \operatorname{ind}_{P_{\gamma}}^{G}\left(M^{*}\right)
$$

for each finite-dimensional $P_{\gamma}$-supermodule $M$.

Example 5.2. For $\lambda \in X_{p}^{+}(T)$, define $V(\lambda):=R^{N} \operatorname{ind}_{B}^{G} \mathfrak{u}\left(w_{0} \lambda\right)$, where $N=$ $\frac{1}{2} n(n-1)$. By Serre duality, $V(\lambda) \cong H^{0}\left(-w_{0} \lambda\right)^{*}$; that is, $V(\lambda)$ is the universal highest weight module of [Brundan and Kleshchev 2003, (6.14), (10.14)]. In particular, $V(\lambda)$ has a unique irreducible quotient isomorphic to $L(\lambda)$, and $\operatorname{ch} V(\lambda)=\operatorname{ch} H^{0}(\lambda)$.

Example 5.3. Let us compute $\operatorname{ch} H^{0}\left(\varepsilon_{1}-\varepsilon_{3}\right)$ for $G=Q(3)$. Let $P_{1}, P_{2}$ denote the minimal parabolic subgroups of $G$ corresponding to the simple roots $\varepsilon_{1}-\varepsilon_{2}$ and $\varepsilon_{2}-\varepsilon_{3}$ respectively. Denote the irreducible $P_{i}$-supermodule of highest weight $\mu$ by $L_{i}(\mu)$. We proceed in steps.

(i) To start with, Serre duality gives $H^{3}\left(\varepsilon_{1}-\varepsilon_{3}\right) \cong H^{0}\left(-\varepsilon_{1}+\varepsilon_{3}\right)^{*}$ which is zero since $-\varepsilon_{1}+\varepsilon_{3} \notin X_{p}^{+}(T)$.

(ii) The $Q(2)$-supermodule $H^{0}\left(\varepsilon_{1}\right)$ is irreducible, so

$$
\operatorname{ind}_{B}^{P_{1}} \mathfrak{u}\left(\varepsilon_{1}-\varepsilon_{3}\right) \cong L_{1}\left(\varepsilon_{1}-\varepsilon_{3}\right) .
$$

Hence,

$$
\left(\operatorname{ind}_{B}^{P_{1}} \mathfrak{u}\left(\varepsilon_{1}-\varepsilon_{3}\right)\right)^{*} \cong L_{1}\left(-\varepsilon_{2}+\varepsilon_{3}\right) \cong \operatorname{ind}_{B}^{P_{1}} \mathfrak{u}\left(-\varepsilon_{2}+\varepsilon_{3}\right)
$$


Applying Serre duality, we deduce that

$$
R^{1} \operatorname{ind}_{B}^{P_{1}} \mathfrak{u}\left(\varepsilon_{2}-\varepsilon_{3}\right) \cong \operatorname{ind}_{B}^{P_{1}} \mathfrak{u}\left(\varepsilon_{1}-\varepsilon_{3}\right) .
$$

Since $R^{i} \operatorname{ind}_{B}^{P_{1}} \mathfrak{u}\left(\varepsilon_{2}-\varepsilon_{3}\right)=0$ for $i \neq 1$, we have

$$
R^{i} \operatorname{ind}_{P_{1}}^{G}\left(R^{1} \operatorname{ind}_{B}^{P_{1}} \mathfrak{u}\left(\varepsilon_{2}-\varepsilon_{3}\right)\right) \cong R^{i+1} \operatorname{ind}_{B}^{G} \mathfrak{u}\left(\varepsilon_{2}-\varepsilon_{3}\right) .
$$

Similarly,

$$
R^{i} \operatorname{ind}_{P_{1}}^{G}\left(\operatorname{ind}_{B}^{P_{1}} \mathfrak{u}\left(\varepsilon_{1}-\varepsilon_{3}\right)\right) \cong R^{i} \operatorname{ind}_{B}^{G} \mathfrak{u}\left(\varepsilon_{1}-\varepsilon_{3}\right) .
$$

So we have shown that

$$
R^{i+1} \operatorname{ind}_{B}^{G} \mathfrak{u}\left(\varepsilon_{2}-\varepsilon_{3}\right) \cong R^{i} \operatorname{ind}_{B}^{G} \mathfrak{u}\left(\varepsilon_{1}-\varepsilon_{3}\right)
$$

for each $i \geq 0$. As in step (i), the left hand side is zero for $i=2$, since $-\varepsilon_{2}+\varepsilon_{3}$ does not lie in $X_{p}^{+}(T)$. Hence, $H^{2}\left(\varepsilon_{1}-\varepsilon_{3}\right)=0$ and $H^{1}\left(\varepsilon_{1}-\varepsilon_{3}\right) \cong R^{2} \operatorname{ind}_{B}^{G} \mathfrak{u}\left(\varepsilon_{2}-\varepsilon_{3}\right)$.

(iii) By Lemma 4.4, the character of the $Q(2)$-supermodule $H^{0}\left(\varepsilon_{1}-\varepsilon_{2}\right)$ is

$$
2 e^{\varepsilon_{1}-\varepsilon_{2}}+4+2 e^{\varepsilon_{2}-\varepsilon_{1}} .
$$

By decomposing the adjoint representation of $Q(2)$, the irreducible $Q(2)$-supermodule $L\left(\varepsilon_{1}-\varepsilon_{2}\right)$ has character $2 e^{\varepsilon_{1}-\varepsilon_{2}}+2+2 e^{\varepsilon_{2}-\varepsilon_{1}}$. Hence, we have the short exact sequence

$$
0 \longrightarrow L_{2}\left(\varepsilon_{2}-\varepsilon_{3}\right) \longrightarrow \operatorname{ind}_{B}^{P_{2}} \mathfrak{u}\left(\varepsilon_{2}-\varepsilon_{3}\right) \longrightarrow C \longrightarrow 0
$$

of $P_{2}$-supermodules, where $C$ has character 2. Dualizing and applying Serre duality gives the short exact sequence

$$
0 \longrightarrow D \longrightarrow R^{1} \operatorname{ind}_{B}^{P_{2}} \mathfrak{u}\left(-\varepsilon_{2}+\varepsilon_{3}\right) \longrightarrow L_{2}\left(\varepsilon_{2}-\varepsilon_{3}\right) \longrightarrow 0
$$

where $D$ has character 2. Considering the resulting long exact sequences gives exact sequences

$R^{2} \operatorname{ind}_{P_{2}}^{G} L_{2}\left(\varepsilon_{2}-\varepsilon_{3}\right) \rightarrow R^{2} \operatorname{ind}_{B}^{G} \mathfrak{u}\left(\varepsilon_{2}-\varepsilon_{3}\right) \rightarrow R^{2} \operatorname{ind}_{P_{2}}^{G} C \rightarrow R^{3} \operatorname{ind}_{P_{2}}^{G} L_{2}\left(\varepsilon_{2}-\varepsilon_{3}\right)=0$ and

$$
0=R^{3} \operatorname{ind}_{B}^{G} \mathfrak{u}\left(-\varepsilon_{2}+\varepsilon_{3}\right) \longrightarrow R^{2} \operatorname{ind}_{P_{2}}^{G} L_{2}\left(\varepsilon_{2}-\varepsilon_{3}\right) \longrightarrow R^{3} \operatorname{ind}_{P_{2}}^{G} D=0 .
$$

Hence $R^{2} \operatorname{ind}_{B}^{G} \mathfrak{u}\left(\varepsilon_{2}-\varepsilon_{3}\right) \cong R^{2} \operatorname{ind}_{P_{2}}^{G} C$. Serre duality implies that the latter has character 2 , so we have shown that $\operatorname{ch} H^{1}\left(\varepsilon_{1}-\varepsilon_{3}\right)=2$.

(iv) Hence, by the definition (10), $\operatorname{ch} H^{0}\left(\varepsilon_{1}-\varepsilon_{3}\right)=E_{\varepsilon_{1}-\varepsilon_{3}}+2$. 
Example 5.4. Continue with $G=Q(3)$ and let $\lambda=4 \varepsilon_{1}+3 \varepsilon_{2}+2 \varepsilon_{3}$. In characteristic $0, H^{0}(\lambda)$ is irreducible by the linkage principle [Brundan and Kleshchev 2003, Theorem 8.10], so its character is known by [Sergeev 1984]: $\operatorname{ch} H^{0}(\lambda)=E_{\lambda}$. But in characteristic 3, the Frobenius twist of determinant gives us a one dimensional $G$-supermodule $\operatorname{det}^{p}$ of character $x^{3\left(\varepsilon_{1}+\varepsilon_{2}+\varepsilon_{3}\right)}$. So by the tensor identity [Brundan and Kleshchev 2003, (6.3)] and the previous example, we have

$$
\operatorname{ch} H^{0}(\lambda)=\operatorname{ch} H^{0}\left(\varepsilon_{1}-\varepsilon_{3}\right) x^{3\left(\varepsilon_{1}+\varepsilon_{2}+\varepsilon_{3}\right)}=E_{\lambda}+2 x^{3\left(\varepsilon_{1}+\varepsilon_{2}+\varepsilon_{3}\right)} .
$$

This example shows that the characters of the induced modules $H^{0}(\lambda)$ are not stable under reduction modulo $p$, even for strict $\lambda$.

\section{Linkage principle for Euler characteristics}

In [Brundan and Kleshchev 2003, §8], we introduced the notion of the residue content $\operatorname{cont}(\lambda)$ of $\lambda \in X(T)$. This is a tuple $\left(c_{0}, c_{1}, \ldots, c_{\ell}\right)$ of integers, where $\ell=(p-1) / 2$ or $\infty$ in case $p=0$. Rather than recall the definition here, we just give one example: let $n=6, \lambda=(9,7,4,0,-5,-8)$ and $p=5$. We represent $\lambda$ pictorially as

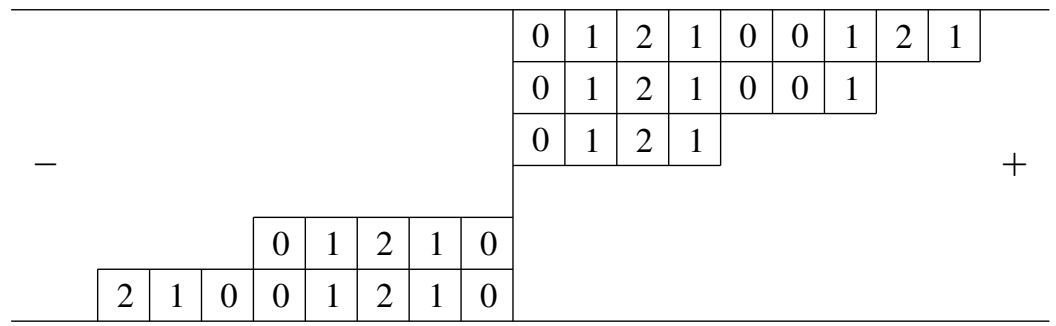

Note in this example that $\ell=2$. The content of $\lambda$ is $(2,4,1), 2$ counting the total number of entries in the diagram equal to 0 (there being 7 in the positive half of the diagram and 5 in the negative half), 4 counting the total number entries equal to 1 and 1 counting the total number of entries equal to 2 .

In Theorem 8.10 of the same reference, we showed that the composition multiplicity $\left[H^{0}(\lambda): L(\mu)\right]$ is zero unless $\operatorname{cont}(\mu)=\operatorname{cont}(\lambda)$. We wish now to show moreover that the decomposition number $d_{\lambda, \mu}$ is zero unless $\operatorname{cont}(\mu)=\operatorname{cont}(\lambda)$. For the proof, we will work in a rather larger category. Recall the definition of the superalgebra of distributions $\operatorname{Dist}(G)$ from [Brundan and Kleshchev 2003, §4]. It was constructed there by reduction modulo $p$ from the Kostant $\mathbb{Z}$-form for the enveloping superalgebra of the Lie superalgebra $\mathfrak{q}(n, \mathbb{C})$, proving that it has a PBW 
basis given by all monomials

$$
\prod_{1 \leq i \neq j \leq n} e_{i, j}^{\left(a_{i, j}\right)}\left(e_{i, j}^{\prime}\right)^{d_{i, j}} \prod_{1 \leq i \leq n}\left(\begin{array}{c}
h_{i} \\
a_{i, i}
\end{array}\right)\left(h_{i}^{\prime}\right)^{d_{i, i}},
$$

for all $a_{i, j} \in \mathbb{Z}_{\geq 0}$ and $d_{i, j} \in\{0,1\}$ (the product being taken in some arbitrary but fixed order). For a $\operatorname{Dist}(G)$-supermodule $M$ and $\lambda=\sum_{i=1}^{n} \lambda_{i} \varepsilon_{i}$, we let

$$
M_{\lambda}=\left\{m \in M \mid\left(\begin{array}{c}
h_{i} \\
r
\end{array}\right) m=\left(\begin{array}{c}
\lambda_{i} \\
r
\end{array}\right) m \text { for all } i=1, \ldots, n, r \geq 0\right\} .
$$

Also let $\operatorname{Dist}\left(B^{+}\right)$be the subalgebra of $\operatorname{Dist}(G)$ generated by $\operatorname{Dist}(H)$ and all $e_{i, j}^{(r)}, e_{i, j}^{\prime}$ for $1 \leq i<j \leq n, r \geq 1$. Let $O_{n}$ be the category of all $\operatorname{Dist}(G)$-supermodules $M$ satisfying the properties

(1) $M$ is locally finite when viewed as a $\operatorname{Dist}\left(B^{+}\right)$-supermodule;

(2) $M=\bigoplus_{\lambda \in X(T)} M_{\lambda}$.

By [Brundan and Kleshchev 2003, Corollary 5.7], we can identify the category of all $G$-supermodules with the full subcategory $\mathscr{C}_{n}$ of $\mathcal{O}_{n}$ consisting of all $M \in \mathcal{O}_{n}$ that are locally finite as $\operatorname{Dist}(G)$-supermodules.

The basic objects in category $\mathrm{O}_{n}$ are the Verma supermodules

$$
M(\lambda):=\operatorname{Dist}(G) \otimes_{\operatorname{Dist}\left(B^{+}\right)} \mathfrak{u}(\lambda)
$$

for each $\lambda \in X(T)$. By standard arguments, $M(\lambda)$ has a unique irreducible quotient denoted $L(\lambda)$, and the supermodules $\{L(\lambda) \mid \lambda \in X(T)\}$ form a complete set of pairwise nonisomorphic irreducibles in category $O_{n}$. Note $L(\lambda)$ is finite-dimensional if and only if $\lambda \in X_{p}^{+}(T)$, when it agrees with the $G$-supermodule denoted $L(\lambda)$ earlier. The character of $M(\lambda)$ is given by

$$
\operatorname{ch} M(\lambda)=x^{\lambda} \prod_{1 \leq i<j \leq n} \frac{1+x_{i}^{-1} x_{j}}{1-x_{i}^{-1} x_{j}} \in \mathbb{Z}\left[x_{1}^{ \pm 1}\right] \llbracket x_{i}^{-1} x_{j} \mid 1 \leq i<j \leq n \rrbracket .
$$

Lemma 6.1. For $\lambda, \mu \in X(T),[M(\lambda): L(\mu)]$ is zero unless $\operatorname{cont}(\lambda)=\operatorname{cont}(\mu)$.

Proof. This follows immediately from [Brundan and Kleshchev 2003, Lemmas 8.4, 8.9].

Lemma 6.2. The series

$$
\prod_{1 \leq i<j \leq n} \frac{1-x_{i}^{-1} x_{j}}{1+x_{i}^{-1} x_{j}} \in \mathbb{Z} \llbracket x_{i}^{-1} x_{j} \mid 1 \leq i<j \leq n \rrbracket
$$

is an infinite linear combination of $x^{\mu}$ 's for $\mu \leq 0$ with $\operatorname{cont}(\mu)=(0, \ldots, 0)$. 
Proof. Working in $\mathbb{Z}\left[x_{1}^{ \pm 1}\right] \llbracket x_{i}^{-1} x_{j} \mid 1 \leq i<j \leq n \rrbracket$, we can write the character 1 of the trivial module as a linear combination of characters of Verma supermodules. In view of (12) and Lemma 6.1, this expression only involves Verma characters of the form $\operatorname{ch} M(\mu)$ for $\mu \leq 0$ with $\operatorname{cont}(\mu)=(0, \ldots, 0)$. Hence,

$$
1=\sum_{\substack{\mu \leq 0 \\ \operatorname{wt}(\mu)=0}}\left(a_{\mu} x^{\mu} \prod_{1 \leq i<j \leq n} \frac{1+x_{i}^{-1} x_{j}}{1-x_{i}^{-1} x_{j}}\right)
$$

for some coefficients $a_{\mu} \in \mathbb{Z}$. The lemma follows.

Now we can prove the main result of the section.

Theorem 6.3. For $\lambda, \mu \in X_{p}^{+}(T), d_{\lambda, \mu}$ is zero unless $\operatorname{cont}(\lambda)=\operatorname{cont}(\mu)$.

Proof. Let $\lambda=\sum_{i=1}^{n} \lambda_{i} \varepsilon_{i} \in X_{p}^{+}(T)$. Working in $\mathbb{Z}\left[x_{1}^{ \pm 1}\right] \llbracket\left[x_{i}^{-1} x_{j} \mid 1 \leq i<j \leq n \rrbracket\right.$, we will show that $E_{\lambda}$ can be written as a (possibly infinite) linear combination of $\operatorname{ch} M(\mu)$ 's for $\mu \in X(T)$ with $\operatorname{cont}(\mu)=\operatorname{cont}(\lambda)$. The theorem follows from this and Lemma 6.1. By Theorem 4.3 and (9), we have

$$
\begin{aligned}
E_{\lambda} & =2^{\lfloor(h(\lambda)+1) / 2\rfloor} \sum_{w \in W^{\gamma(\lambda)}} w\left(x^{\lambda} \prod_{\substack{1 \leq i<j \leq n \\
\lambda_{i}=\lambda_{j}}} \frac{1-x_{i}^{-1} x_{j}}{1+x_{i}^{-1} x_{j}} \prod_{1 \leq i<j \leq n} \frac{1+x_{i}^{-1} x_{j}}{1-x_{i}^{-1} x_{j}}\right) \\
& =2^{\lfloor(h(\lambda)+1) / 2\rfloor} \sum_{w \in W^{\gamma}(\lambda)}(-1)^{\ell(w)} x^{x^{w \lambda} \prod_{\substack{1 \leq i<j \leq n \\
\lambda_{i}=\lambda_{j}}} \frac{1-x_{w i}^{-1} x_{w j}}{1+x_{w i}^{-1} x_{w j}}} \prod_{1 \leq i<j \leq n} \frac{1+x_{i}^{-1} x_{j}}{1-x_{i}^{-1} x_{j}} .
\end{aligned}
$$

By Lemma 6.2, the term marked by braces is a (possibly infinite) linear combination of $x^{\mu}$ 's for $\mu \leq w \lambda$ with $\operatorname{cont}(\mu)=\operatorname{cont}(w \lambda)=\operatorname{cont}(\lambda)$. Hence recalling (12), $E_{\lambda}$ is a (possibly infinite) linear combination of $\operatorname{ch} M(\mu)$ 's for $\mu \in X(T)$ with $\operatorname{cont}(\mu)=\operatorname{cont}(\lambda)$.

Supplement: Some decomposition matrices for polynomial representations in characteristic $p=3$, for $n$ large.

\begin{tabular}{c|cc}
$d=3$ & $(21)$ & $(3)$ \\
\hline$(21)$ & 1 & 0 \\
$(3)$ & 1 & 1
\end{tabular}

\begin{tabular}{c|cc}
$d=4$ & $(31)$ & $(4)$ \\
\hline$(31)$ & 1 & 0 \\
$(4)$ & 1 & 1
\end{tabular}

\begin{tabular}{c|ccc}
$d=5$ & $(32)$ & $(41)$ & $(5)$ \\
\hline$(32)$ & 1 & 0 & 0 \\
$(41)$ & 0 & 1 & 0 \\
$(5)$ & 1 & 0 & 1
\end{tabular}




\begin{tabular}{c|cccccc|ccccccc}
$d=6$ & $(321)$ & $\left(3^{2}\right)$ & $(42)$ & $(51)$ & $(6)$ & & $d=7$ & $\left(3^{2} 1\right)$ & $(421)$ & $(43)$ & $(52)$ & $(61)$ & $(7)$ \\
\hline$(321)$ & 1 & 0 & 0 & 0 & 0 & & $\left(3^{2} 1\right)$ & 1 & 0 & 0 & 0 & 0 & 0 \\
$\left(3^{2}\right)$ & 1 & 1 & 0 & 0 & 0 & & $(421)$ & 2 & 1 & 0 & 0 & 0 & 0 \\
$(42)$ & 2 & 2 & 1 & 0 & 0 & & $(43)$ & 1 & 1 & 1 & 0 & 0 & 0 \\
$(51)$ & 1 & 2 & 1 & 1 & 0 & & $(52)$ & 0 & 0 & 0 & 1 & 0 & 0 \\
$(6)$ & 1 & 1 & 0 & 1 & 1 & & $(61)$ & 1 & 1 & 1 & 0 & 1 & 0 \\
& & & & & & $(7)$ & 1 & 0 & 0 & 0 & 1 & 1
\end{tabular}

\begin{tabular}{c|ccccccc}
$d=8$ & $\left(3^{2} 2\right)$ & $(431)$ & $(521)$ & $(53)$ & $(62)$ & $(71)$ & $(8)$ \\
\hline$\left(3^{2} 2\right)$ & 1 & 0 & 0 & 0 & 0 & 0 & 0 \\
$(431)$ & 0 & 1 & 0 & 0 & 0 & 0 & 0 \\
$(521)$ & 2 & 0 & 1 & 0 & 0 & 0 & 0 \\
$(53)$ & 1 & 0 & 1 & 1 & 0 & 0 & 0 \\
$(62)$ & 1 & 0 & 1 & 1 & 1 & 0 & 0 \\
$(71)$ & 0 & 1 & 0 & 0 & 0 & 1 & 0 \\
$(8)$ & 1 & 0 & 0 & 0 & 1 & 0 & 1
\end{tabular}

\begin{tabular}{c|cccccccccc}
$d=9$ & $\left(3^{2} 21\right)$ & $\left(3^{3}\right)$ & $(432)$ & $(531)$ & $(54)$ & $(621)$ & $(63)$ & $(72)$ & $(81)$ & $(9)$ \\
\hline$\left(3^{2} 21\right)$ & 1 & 0 & 0 & 0 & 0 & 0 & 0 & 0 & 0 & 0 \\
$\left(3^{3}\right)$ & 1 & 1 & 0 & 0 & 0 & 0 & 0 & 0 & 0 & 0 \\
$(432)$ & 0 & 0 & 1 & 0 & 0 & 0 & 0 & 0 & 0 & 0 \\
$(531)$ & 2 & 2 & 1 & 1 & 0 & 0 & 0 & 0 & 0 & 0 \\
$(54)$ & 1 & 2 & 0 & 1 & 1 & 0 & 0 & 0 & 0 & 0 \\
$(621)$ & 2 & 2 & 0 & 1 & 0 & 1 & 0 & 0 & 0 & 0 \\
$(63)$ & 1 & 3 & 0 & 1 & 1 & 1 & 1 & 0 & 0 & 0 \\
$(72)$ & 1 & 6 & 1 & 1 & 1 & 2 & 2 & 1 & 0 & 0 \\
$(81)$ & 1 & 4 & 1 & 0 & 0 & 1 & 2 & 1 & 1 & 0 \\
$(9)$ & 1 & 2 & 0 & 0 & 0 & 1 & 2 & 0 & 1 & 1
\end{tabular}

\begin{tabular}{c|cccccccccccc}
$d=10$ & $\left(3^{3} 1\right)$ & $(4321)$ & $\left(43^{2}\right)$ & $(532)$ & $(541)$ & $(631)$ & $(64)$ & $(721)$ & $(73)$ & $(82)$ & $(91)$ & $(10)$ \\
\hline$\left(3^{3} 1\right)$ & 1 & 0 & 0 & 0 & 0 & 0 & 0 & 0 & 0 & 0 & 0 & 0 \\
$(4321)$ & 0 & 1 & 0 & 0 & 0 & 0 & 0 & 0 & 0 & 0 & 0 & 0 \\
$\left(43^{2}\right)$ & 1 & 1 & 1 & 0 & 0 & 0 & 0 & 0 & 0 & 0 & 0 & 0 \\
$(532)$ & 0 & 0 & 0 & 1 & 0 & 0 & 0 & 0 & 0 & 0 & 0 & 0 \\
$(541)$ & 2 & 2 & 2 & 0 & 1 & 0 & 0 & 0 & 0 & 0 & 0 & 0 \\
$(631)$ & 2 & 1 & 1 & 0 & 1 & 1 & 0 & 0 & 0 & 0 & 0 & 0 \\
$(64)$ & 1 & 0 & 1 & 0 & 1 & 1 & 1 & 0 & 0 & 0 & 0 & 0 \\
$(721)$ & 2 & 1 & 2 & 0 & 1 & 2 & 0 & 1 & 0 & 0 & 0 & 0 \\
$(73)$ & 1 & 1 & 3 & 0 & 1 & 2 & 1 & 1 & 1 & 0 & 0 & 0 \\
$(82)$ & 0 & 0 & 0 & 1 & 0 & 0 & 0 & 0 & 0 & 1 & 0 & 0 \\
$(91)$ & 1 & 1 & 2 & 0 & 0 & 1 & 0 & 1 & 1 & 0 & 1 & 0 \\
$(10)$ & 1 & 0 & 0 & 0 & 0 & 1 & 0 & 0 & 0 & 0 & 1 & 1
\end{tabular}




\begin{tabular}{c|ccccccccccccccc}
$d=11$ & $3^{3} 2$ & $43^{2} 1$ & 5321 & $53^{2}$ & 542 & 632 & 641 & 65 & 731 & 74 & 821 & 83 & 92 & 10,1 & 11 \\
\hline $3^{3} 2$ & 1 & 0 & 0 & 0 & 0 & 0 & 0 & 0 & 0 & 0 & 0 & 0 & 0 & 0 & 0 \\
$43^{2} 1$ & 0 & 1 & 0 & 0 & 0 & 0 & 0 & 0 & 0 & 0 & 0 & 0 & 0 & 0 & 0 \\
5321 & 0 & 0 & 1 & 0 & 0 & 0 & 0 & 0 & 0 & 0 & 0 & 0 & 0 & 0 & 0 \\
$53^{2}$ & 1 & 0 & 1 & 1 & 0 & 0 & 0 & 0 & 0 & 0 & 0 & 0 & 0 & 0 & 0 \\
542 & 2 & 0 & 2 & 2 & 1 & 0 & 0 & 0 & 0 & 0 & 0 & 0 & 0 & 0 & 0 \\
632 & 2 & 0 & 1 & 1 & 1 & 1 & 0 & 0 & 0 & 0 & 0 & 0 & 0 & 0 & 0 \\
641 & 0 & 1 & 0 & 0 & 0 & 0 & 1 & 0 & 0 & 0 & 0 & 0 & 0 & 0 & 0 \\
65 & 1 & 0 & 0 & 1 & 1 & 1 & 0 & 1 & 0 & 0 & 0 & 0 & 0 & 0 & 0 \\
731 & 0 & 1 & 0 & 0 & 0 & 0 & 1 & 0 & 1 & 0 & 0 & 0 & 0 & 0 & 0 \\
74 & 0 & 0 & 0 & 0 & 0 & 0 & 1 & 0 & 1 & 1 & 0 & 0 & 0 & 0 & 0 \\
821 & 2 & 0 & 1 & 2 & 1 & 2 & 0 & 0 & 0 & 0 & 1 & 0 & 0 & 0 & 0 \\
83 & 1 & 0 & 1 & 3 & 1 & 2 & 0 & 1 & 0 & 0 & 1 & 1 & 0 & 0 & 0 \\
92 & 1 & 0 & 1 & 2 & 0 & 1 & 0 & 0 & 0 & 0 & 1 & 1 & 1 & 0 & 0 \\
10,1 & 0 & 1 & 0 & 0 & 0 & 0 & 0 & 0 & 1 & 0 & 0 & 0 & 0 & 1 & 0 \\
11 & 1 & 0 & 0 & 0 & 0 & 1 & 0 & 0 & 0 & 0 & 0 & 1 & 1 & 0 & 1
\end{tabular}

\begin{tabular}{c|cccccccccccccccccccccccc}
$d=12$ & $3^{3} 21$ & $3^{4}$ & $43^{2}$ & $53^{2} 1$ & 5421 & 543 & 6321 & $63^{2}$ & 642 & 651 & $6^{2}$ & 732 & 741 & 75 & 831 & 84 & 921 & 93 & 10,2 & 11,1 & 12 \\
\hline $3^{3} 21$ & 1 & 0 & 0 & 0 & 0 & 0 & 0 & 0 & 0 & 0 & 0 & 0 & 0 & 0 & 0 & 0 & 0 & 0 & 0 & 0 & 0 \\
$3^{4}$ & 1 & 1 & 0 & 0 & 0 & 0 & 0 & 0 & 0 & 0 & 0 & 0 & 0 & 0 & 0 & 0 & 0 & 0 & 0 & 0 & 0 \\
$43^{2} 2$ & 2 & 2 & 1 & 0 & 0 & 0 & 0 & 0 & 0 & 0 & 0 & 0 & 0 & 0 & 0 & 0 & 0 & 0 & 0 & 0 & 0 \\
$53^{2} 1$ & 0 & 0 & 1 & 1 & 0 & 0 & 0 & 0 & 0 & 0 & 0 & 0 & 0 & 0 & 0 & 0 & 0 & 0 & 0 & 0 & 0 \\
5421 & 0 & 0 & 0 & 2 & 1 & 0 & 0 & 0 & 0 & 0 & 0 & 0 & 0 & 0 & 0 & 0 & 0 & 0 & 0 & 0 & 0 \\
543 & 1 & 2 & 0 & 1 & 1 & 1 & 0 & 0 & 0 & 0 & 0 & 0 & 0 & 0 & 0 & 0 & 0 & 0 & 0 & 0 & 0 \\
6321 & 1 & 2 & 0 & 1 & 1 & 0 & 1 & 0 & 0 & 0 & 0 & 0 & 0 & 0 & 0 & 0 & 0 & 0 & 0 & 0 & 0 \\
$63^{2}$ & 2 & 3 & 0 & 1 & 1 & 1 & 1 & 1 & 0 & 0 & 0 & 0 & 0 & 0 & 0 & 0 & 0 & 0 & 0 & 0 & 0 \\
642 & 4 & 8 & 1 & 2 & 3 & 2 & 2 & 2 & 1 & 0 & 0 & 0 & 0 & 0 & 0 & 0 & 0 & 0 & 0 & 0 & 0 \\
651 & 1 & 4 & 1 & 0 & 1 & 1 & 1 & 2 & 1 & 1 & 0 & 0 & 0 & 0 & 0 & 0 & 0 & 0 & 0 & 0 & 0 \\
$6^{2}$ & 1 & 4 & 0 & 0 & 1 & 1 & 1 & 1 & 0 & 1 & 1 & 0 & 0 & 0 & 0 & 0 & 0 & 0 & 0 & 0 & 0 \\
732 & 3 & 6 & 1 & 1 & 2 & 1 & 2 & 2 & 1 & 0 & 0 & 1 & 0 & 0 & 0 & 0 & 0 & 0 & 0 & 0 & 0 \\
741 & 0 & 0 & 0 & 0 & 0 & 0 & 0 & 0 & 0 & 0 & 0 & 0 & 1 & 0 & 0 & 0 & 0 & 0 & 0 & 0 & 0 \\
75 & 2 & 6 & 0 & 0 & 2 & 2 & 2 & 2 & 1 & 2 & 0 & 1 & 0 & 1 & 0 & 0 & 0 & 0 & 0 & 0 & 0 \\
831 & 2 & 6 & 1 & 1 & 2 & 2 & 3 & 2 & 1 & 1 & 0 & 1 & 0 & 0 & 1 & 0 & 0 & 0 & 0 & 0 & 0 \\
84 & 1 & 4 & 0 & 1 & 2 & 3 & 3 & 2 & 1 & 2 & 0 & 1 & 0 & 1 & 1 & 1 & 0 & 0 & 0 & 0 & 0 \\
921 & 2 & 2 & 0 & 1 & 1 & 1 & 2 & 0 & 0 & 0 & 0 & 0 & 0 & 0 & 1 & 0 & 1 & 0 & 0 & 0 & 0 \\
93 & 1 & 3 & 0 & 1 & 1 & 2 & 2 & 1 & 0 & 1 & 1 & 0 & 0 & 0 & 1 & 1 & 1 & 1 & 0 & 0 & 0 \\
10,2 & 1 & 0 & 1 & 1 & 0 & 1 & 1 & 0 & 0 & 0 & 0 & 1 & 0 & 0 & 1 & 1 & 2 & 0 & 1 & 0 & 0 \\
11,1 & 1 & 2 & 1 & 0 & 0 & 0 & 1 & 0 & 0 & 0 & 0 & 1 & 0 & 0 & 0 & 0 & 1 & 0 & 1 & 1 & 0 \\
12 & 1 & 1 & 0 & 0 & 0 & 0 & 1 & 1 & 0 & 0 & 1 & 0 & 0 & 0 & 0 & 0 & 1 & 1 & 0 & 1 & 1
\end{tabular}

\section{References}

[Brundan 2004] J. Brundan, "Kazhdan-Lusztig polynomials and character formulae for the Lie superalgebra q(n)", Adv. Math. 182:1 (2004), 28-77. MR 2004m:17018 Zbl 1048.17003

[Brundan and Kleshchev 2002] J. Brundan and A. Kleshchev, "Projective representations of symmetric groups via Sergeev duality”, Math. Z. 239:1 (2002), 27-68. MR 2003b:20018 Zbl 1029.20008 
[Brundan and Kleshchev 2003] J. Brundan and A. Kleshchev, "Modular representations of the supergroup $Q(n)$. I”, J. Algebra 260:1 (2003), 64-98. MR 2004f:20081 Zbl 1027.17004

[Chriss and Ginzburg 1997] N. Chriss and V. Ginzburg, Representation theory and complex geometry, Birkhäuser, Boston, 1997. MR 98i:22021 Zbl 0879.22001

[Cline et al. 1983] E. Cline, B. Parshall, and L. Scott, "A Mackey imprimitivity theory for algebraic groups", Math. Z. 182:4 (1983), 447-471. MR 84j:14046 Zbl 0537.14029

[Demazure 1969/1970] M. Demazure, "Sur la formule des caractères de H. Weyl", Invent. Math. 9 (1969/1970), 249-252. MR 44 \#5321 Zbl 0189.21403

[Demazure and Gabriel 1970] M. Demazure and P. Gabriel, Groupes algébriques, vol. 1: Géométrie algébrique, généralités, groupes commutatifs, Masson, 1970. MR 46 \#1800 Zbl 0203.23401

[Hartshorne 1977] R. Hartshorne, Algebraic geometry, Graduate Texts in Math. 52, Springer, New York, 1977. MR 57 \#3116 Zbl 0367.14001

[Jantzen 1987] J. C. Jantzen, Representations of algebraic groups, Pure and Applied Mathematics 131, Academic Press, Boston, 1987. 2nd ed., Amer. Math. Soc., Providence, 2003. MR 89c:20001 Zbl 1034.20041

[Leclerc and Thibon 1997] B. Leclerc and J.-Y. Thibon, " $q$-deformed Fock spaces and modular representations of spin symmetric groups”, J. Phys. A 30:17 (1997), 6163-6176. MR 99c:17029 Zbl 1039.17509

[Macdonald 1995] I. G. Macdonald, Symmetric functions and Hall polynomials, 2nd ed., Oxford University Press, New York, 1995. MR 96h:05207 Zbl 0899.05068

[Manin 1991] Y. I. Manin, Topics in noncommutative geometry, Princeton University Press, Princeton, NJ, 1991. MR 92k:58024 Zbl 0724.17007

[Manin 1997] Y. I. Manin, Gauge field theory and complex geometry, 2nd ed., Grundlehren der Math. Wissenschaften 289, Springer, Berlin, 1997. MR 99e:32001 Zbl 0641.53001

[Mumford and Fogarty 1982] D. Mumford and J. Fogarty, Geometric invariant theory, 2nd ed., Ergebnisse der Mathematik 34, Springer, Berlin, 1982. MR 86a:14006 Zbl 0504.14008

[Penkov 1986] I. B. Penkov, "Characters of typical irreducible finite-dimensional q(n)-modules", Funktsional. Anal. i Prilozhen. 20:1 (1986), 37-45. In Russian; translated in Func. Anal. Appl. 20 (1986), 30-37. MR 87j:17033 Zbl 0595.17003

[Penkov 1988] I. B. Penkov, "Borel-Weil-Bott theory for classical Lie supergroups", pp. 71-124 in Современные проблемы математики, vol. 32, Vsesoyuz. Inst. Nauchn. i Tekhn. Inform., Moscow, 1988. In Russian; translated in J. Soviet Math. 51:1 (1990), 2108-2140. MR 90f:22018 Zbl 0734.17004

[Penkov and Serganova 1989] I. Penkov and V. Serganova, "Cohomology of $G / P$ for classical complex Lie supergroups $G$ and characters of some atypical $G$-modules", Ann. Inst. Fourier (Grenoble) 39:4 (1989), 845-873. MR 91k:14036 Zbl 0667.14023

[Penkov and Serganova 1997a] I. Penkov and V. Serganova, "Characters of finite-dimensional irreducible q(n)-modules”, Lett. Math. Phys. 40:2 (1997), 147-158. MR 98i:17011 Zbl 0892.17006

[Penkov and Serganova 1997b] I. Penkov and V. Serganova, "Characters of irreducible $G$-modules and cohomology of $G / P$ for the Lie supergroup $G=Q(N)$ ", J. Math. Sci. (New York) 84:5 (1997), 1382-1412. MR 98i:17010 Zbl 0920.17003

[Sergeev 1984] A. N. Sergeev, "Tensor algebra of the identity representation as a module over the Lie superalgebras GL( $n, m)$ and $Q(n)$ ", Mat. Sb. (N.S.) 123:3 (1984), 422-430. In Russian; translated in Math. USSR Sbornik 51 (1985), 419-427. MR 85h:17010 Zbl 0573.17002 
[Voronov et al. 1988] A. A. Voronov, Y. I. Manin, and I. B. Penkov, "Elements of supergeometry”, pp. 3-25 in Современные проблемы математики, vol. 32, Vsesoyuz. Inst. Nauchn. i Tekhn. Inform., Moscow, 1988. In Russian; translated in J. Soviet Math. 51:1 (1990), 2069-2083. MR 90c:58016 Zbl 0736.58004

Received July 20, 2004.

JONATHAN BRUNDAN

DEPARTMENT OF MATHEMATICS

UNIVERSITY OF OREGON

EUGENE, OR 97403

UNITED STATES

brundan@darkwing.uoregon.edu

http://darkwing.uoregon.edu/ brundan 